Interfaces and Free Boundaries 18 (2016), 317-353

DOI $10.4171 / \mathrm{IFB} / 366$

\title{
A Hopf-Lax formula for the time evolution of the level-set equation and a new approach to shape sensitivity analysis
}

\author{
DANIEL KRAFT \\ University of Graz, Institute of Mathematics, NAWI Graz, Universitätsplatz 3, 8010 Graz, Austria \\ E-mail: daniel.kraft@uni-graz.at
}

[Received 12 May 2015 and in revised form 26 February 2016]

\begin{abstract}
The level-set method is used in many different applications to describe the propagation of shapes and domains. When scalar speed fields are used to encode the desired shape evolution, this leads to the classical level-set equation. We present a concise Hopf-Lax representation formula that can be used to characterise the evolved domains at arbitrary times. This result is also applicable for the case of speed fields without a fixed sign, even though the level-set equation has a non-convex Hamiltonian in these situations. The representation formula is based on the same idea that underpins the FastMarching Method, and it provides a strong theoretical justification for a generalised Composite FastMarching method.

Based on our Hopf-Lax formula, we are also able to present new theoretical results. In particular, we show non-fattening of the zero level set in a measure-theoretic sense, derive a very general shapesensitivity calculus that does not require the usual regularity assumptions on the domains, prove optimal Lipschitz constants for the evolved level-set function and discuss the effect of perturbations in both the speed field and the initial geometry.
\end{abstract}

2010 Mathematics Subject Classification: Primary 49L25; Secondary 49Q10.

Keywords: Level-set method, shape optimisation, Hopf-Lax formula, viscosity solutions, nonfattening, shape-sensitivity analysis.

\section{Introduction}

Many problem classes in applied mathematics require the manipulation of shapes and geometries (shape optimisation being an obvious one, but also free-boundary problems fall into this category). For this, it is necessary to "encode" the geometric information in a suitable way. Very thorough general discussions of this topic can be found in the classical books [13], [23] and [39]. For this paper, we focus on the level-set method. It was introduced in [30] by Osher and Sethian. Today, there exists a vast literature about it, covering various aspects. A general introduction can be found in [34]. For selected theoretical results, we would like to highlight [20] and [5]. Applications of the level-set method to concrete problems can be found, for instance, in [9], [37], [14] and [6].

The basic idea in the level-set framework is to introduce an auxiliary level-set function $\phi: \mathbb{R}^{n} \rightarrow$ $\mathbb{R}$ to describe an open set $\Omega \subset \mathbb{R}^{n}$. The domain

$$
\Omega=\phi^{-1}((-\infty, 0))
$$

is given as the sub-zero level set of $\phi$. This set is obviously open if $\phi$ is continuous. Of course, many different level-set functions can describe a single open set. A possible choice for the level-set function of some given $\Omega$ is its signed distance function (see Chapter 5 of [13]; also note [36], which 


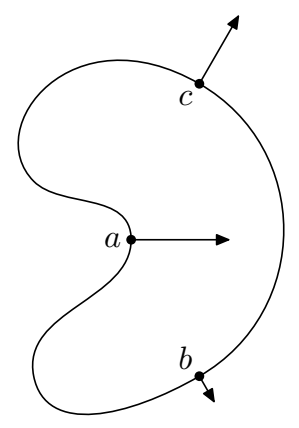

FIG. 1: Illustration of the speed method. In the shown situation, $F(a)<0<F(b)<F(c)$.

formulates the shape-evolution problem in terms of the signed distance function). Since this works for arbitrary open sets, we immediately see that even Lipschitz continuity of the level-set function implies no regularity of $\Omega$. (Throughout this work, we will concentrate on Lipschitz continuous level-set functions. Note that higher-order regularity of a level-set function does, in fact, imply boundary regularity of the described domain. See, for instance, Theorem 4.2 on page 77 of [13].) The other way round, however, this also means that the level-set method is very flexible and can describe a wide range of shapes.

In order to describe not only geometries themselves but also changes to them, let us consider for the moment shape deformations by the classical speed method: Given a scalar speed field $F$ : $\mathbb{R}^{n} \rightarrow \mathbb{R}$, we move the boundary of $\Omega$ in normal direction according to this speed field. Positive speed corresponds to outward movement of the boundary (growth of $\Omega$ ), while negative speed leads to local shrinking. This is illustrated in Figure 1. In terms of the level-set function, the corresponding time evolution is described by the level-set equation

$$
\phi_{t}(x, t)+F(x)|\nabla \phi(x, t)|=0 \text { in } \mathbb{R}^{n} \times(0, \infty), \quad \phi(x, 0)=\phi_{0}(x) \text { for } x \in \mathbb{R}^{n} \text { and } t=0
$$

as introduced in [30]. Throughout this work we will assume that $F$ is Lipschitz continuous with Lipschitz constant $L_{F}$ and that it has compact support. Some of our results could be proven without these assumptions, but we make them nevertheless for simplicity. They are easy to justify in many concrete situations and necessary for the more interesting conclusions anyway. The function $\phi_{0}$ shall also be Lipschitz continuous, and we denote its Lipschitz constant by $L_{\phi_{0}}$. As mentioned already, a canonical choice of $\phi_{0}$ is the signed distance function of an initial (bounded) open set $\Omega_{0}$. With this choice, $L_{\phi_{0}}=1$.

The main result of this paper will be a Hopf-Lax representation formula for the time evolution of both $\phi(\cdot, t)$ itself and the corresponding evolving set $\Omega_{t}$. Similar formulas were first investigated in [2], and are derived, for instance, in [19]. In this paper, however, we also make use of our representation formulas to derive new theoretical results useful for the analysis of shape evolutions in the context of optimisation. Section 2 recalls and introduces some necessary properties of the level-set equation (1) and its solution $\phi$. Section 3 discusses the Eikonal equation and shortest paths induced by a speed field $F$. Based on these foundations, we can then introduce the Hopf-Lax formula itself in Section 4. Theorem 5 and Corollary 1 are the main theorems. They allow us to analyse the geometric evolution on a more abstract level, without the need to work with the level-set 
equation itself. This is a very useful simplification, and we will draw interesting further conclusions from it in Section 5.

Note that this paper is largely based on the initial chapters of [27], where some additional details can be found that fall outside of the scope of this work. Somewhat related to our results is the Generalised Fast Marching Method described in [8]. However, note that [8] is focused solely on the algorithmic analysis of the introduced numerical method. It does not state a general-purpose HopfLax formula for the time evolution. We, on the other hand, would like to focus on the representation formula and the conclusions it enables from a theoretical point of view. Nevertheless, it is also important to remark that our results give a theoretical justification for the Fast Marching Method (see [33] and Chapter 8 of [34]), and also generalise it to our Composite Fast-Marching method that is able to handle sign changes in the speed field. For an application of the latter to PDE-constrained shape optimisation, see [25]. Our numerical implementation is available together with additional tools for the level-set method as free software in the level-set package [26] for GNU Octave [15].

\section{Preliminaries about the Level-Set Equation}

Since the proper solution concept for the level-set equation (1) is that of viscosity solutions, we quickly recall their definition. More details can be found, for instance, in [11] and [12].

Definition 1 Let $D=\mathbb{R}^{n} \times(0, \infty)$ be the open space-time cylinder, $\phi: D \rightarrow \mathbb{R}$ and $(x, t) \in D$. Then $J^{1+} \phi(x, t)$ is the set of all $(p, a) \in \mathbb{R}^{n} \times \mathbb{R}$ such that

$$
\phi(y, s) \leqslant \phi(x, t)+a(s-t)+p \cdot(y-x)+o(|s-t|+|y-x|)
$$

as $(y, s) \rightarrow(x, t)$ in $D$. Similarly, $(p, a) \in J^{1-} \phi(x, t)$ if and only if

$$
\phi(y, s) \geqslant \phi(x, t)+a(s-t)+p \cdot(y-x)+o(|s-t|+|y-x|)
$$

for $(y, s) \rightarrow(x, t) . J^{1 \pm} \phi(x, t)$ are called the first-order parabolic semijets of $\phi$ at $(x, t)$. Note that $J^{1-} \phi(x, t)$ is often also called subdifferential of $\phi$ at $(x, t)$.

DEFINITION 2 Let $F$ and $\phi_{0}$ be given. We say that $\phi: \mathbb{R}^{n} \times[0, \infty) \rightarrow \mathbb{R}$ is a viscosity subsolution of (1) for the given data if $\phi$ is upper semi-continuous, $\phi(\cdot, 0) \leqslant \phi_{0}$ on $\mathbb{R}^{n}$ and

$$
a+F(x)|p| \leqslant 0
$$

for each $(x, t) \in D$ and $(p, a) \in J^{1+} \phi(x, t)$. Similarly, $\phi$ is a viscosity supersolution if $\phi$ is lower semi-continuous, $\phi(\cdot, 0) \geqslant \phi_{0}$ and $a+F(x)|p| \geqslant 0$ for all $(p, a) \in J^{1-} \phi(x, t)$.

The function $\phi$ solves (1) in the viscosity sense if it is both a viscosity sub- and supersolution. Note that this implies, in particular, that $\phi$ is continuous and that $\phi(x, 0)=\phi_{0}(x)$ for all $x \in \mathbb{R}^{n}$.

The following result, which states the existence of a unique viscosity solution to (1) as well as the so-called comparison principle, is well-known:

Theorem 1 For given $F$ and $\phi_{0}$, there exists a unique viscosity solution $\phi: \mathbb{R}^{n} \times[0, \infty) \rightarrow \mathbb{R}$ of (1).

Furthermore, if $\phi_{1}$ and $\phi_{2}$ are viscosity sub- and supersolutions to (1), respectively, with $\phi_{1}(x, 0) \leqslant \phi_{2}(x, 0)$ for all $x \in \mathbb{R}^{n}$, then $\phi_{1} \leqslant \phi_{2}$ pointwise on $\mathbb{R}^{n} \times[0, \infty)$. 
See, for instance, [20] for a thorough treatment of this and related results. The second-order case was first proven in [10] (Theorem 4.1) and [18] (Theorem 3.2).

We will now proceed to deduce several useful consequences of this solution concept for (1). As our first result, we show an intuitively trivial but still important fact: If the speed vanishes everywhere, then the solution of (1) is constant in time.

Lemma 1 Let $\phi$ solve (1) for $F=0$ and some initial function $\phi_{0}$. Then $\phi(x, t)=\phi_{0}(x)$ for all $x \in \mathbb{R}^{n}$ and all times $t \geqslant 0$.

Proof. We have to show that $\phi(x, t)=\phi_{0}(x)$ is indeed a solution of (1) with $F=0$. It is clear that the initial condition is satisfied. Let $(p, a) \in J^{1+} \phi(x, t)$ for some $(x, t) \in D$. Then

$$
\phi(y, s)-\phi(x, t) \leqslant a(s-t)+p \cdot(y-x)+o(|s-t|+|y-x|)
$$

for all $y \in \mathbb{R}^{n}$ and $s>0$ by the definition of $J^{1+} \phi(x, t)$. Consider, in particular, $y=x$ and a sequence $s_{k} \rightarrow t$. The left-hand side of (2) vanishes since $\phi$ is constant in time, so that we can re-arrange the relation to read

$$
0 \leqslant a \cdot \frac{s_{k}-t}{\left|s_{k}-t\right|}+\frac{o\left(\left|s_{k}-t\right|\right)}{\left|s_{k}-t\right|} .
$$

For $s_{k}$ converging to $t$ from above, this gives $0 \leqslant a$ in the limit. For $s_{k} \rightarrow t^{-}$from below, it follows that $0 \leqslant-a$, thus $a=0$ must necessarily hold. Hence $a+F(x)|p|=0 \leqslant 0$ is satisfied, and $\phi$ is indeed a viscosity subsolution of (1). In the same way, one can also show that it is a supersolution.

The comparison principle (second part of Theorem 1) implies that the solution is monotone with respect to the speed:

Lemma 2 Let $F_{1} \geqslant F_{2}$ in $\mathbb{R}^{n}$, and let $\phi_{1}$ and $\phi_{2}$ be solutions to (1) for $F=F_{1}$ and $F=F_{2}$, respectively, with initial conditions $\phi_{1}(x, 0)=\phi_{1,0}(x)$ and $\phi_{2}(x, 0)=\phi_{2,0}(x)$. If $\phi_{1,0} \leqslant \phi_{2,0}$ on $\mathbb{R}^{n}$, then $\phi_{1} \leqslant \phi_{2}$ on the whole of $\mathbb{R}^{n} \times[0, \infty)$.

Proof. We shall show that $\phi_{1}$ is a viscosity subsolution to (1) also with speed $F=F_{2}$. Then Theorem 1 implies the claim. Let $(p, a) \in J^{1+} \phi_{1}(x, t)$ for some $(x, t) \in D$. Then

$$
a+F_{2}(x)|p| \leqslant a+F_{1}(x)|p| \leqslant 0
$$

since $\phi_{1}$ is a solution to the equation with $F=F_{1}$. Thus it is, indeed, also a subsolution with $F=F_{2}$.

Lemma 3 Let $F \geqslant 0$ and $\phi$ solve (1). Then for each $x \in \mathbb{R}^{n}, \phi(x, \cdot)$ is decreasing on $[0, \infty)$. If $F \leqslant 0$ instead, then $\phi(x, \cdot)$ is increasing in time.

Proof. Let $F \geqslant 0$ and $s \geqslant 0$ be given. We have to show $\phi(x, s) \geqslant \phi(x, t)$ for all $x \in \mathbb{R}^{n}$ and $t>s$. If $s>0$, we can shift the initial time to $s$ and use $\phi(\cdot, s)$ as initial data, so assume $s=0$ without loss of generality. By Lemma 1 , we know that $\tilde{\phi}(x, t)=\phi(x, 0)$ solves (1) with $\tilde{F}=0$. Since $F \geqslant 0=\tilde{F}$, Lemma 2 implies that $\phi(x, t) \leqslant \tilde{\phi}(x, t)=\phi(x, 0)$, which finishes the proof. For the case $F \leqslant 0$, a similar argument can be applied. 
Note that, due to the concept of viscosity solutions we use, (1) has no time-reversal symmetry. However, there exists an important symmetry property with respect to sign changes in $F$ and $\phi$, which will be useful later:

Lemma 4 Let $\phi$ be the solution of (1) for some $F$ and $\phi_{0}$. Then $-\phi$ solves the equation for $-F$ and with initial data $-\phi_{0}$.

Proof. The initial condition is obviously satisfied. Let $(p, a) \in J^{1+}(-\phi)(x, t)$, and denote the generic error term for simplicity by $r=o(|s-t|+|y-x|)$. Then it holds that

$$
\begin{gathered}
(-\phi)(y, s) \leqslant(-\phi)(x, t)+a(s-t)+p \cdot(y-x)+r, \\
\phi(y, s) \geqslant \phi(x, t)-a(s-t)-p \cdot(y-x)+r .
\end{gathered}
$$

Hence, $(-p,-a) \in J^{1-} \phi(x, t)$. Since $\phi$ is a supersolution of (1), this implies that

$$
-a+F(x)|-p|=-a+F(x)|p| \geqslant 0 \Leftrightarrow a-F(x)|p| \leqslant 0 .
$$

Thus $-\phi$ is a subsolution when the speed is $-F$. By the same argument, one can show that $-\phi$ is a supersolution in this case as well.

We are now able to show an interesting result that allows us to reduce the general problem to the case where $F \geqslant 0$ or $F \leqslant 0$ throughout the domain. The latter case can be reduced itself to $F \geqslant 0$ by Lemma 4. Hence, we can restrict ourselves to the consideration of $F \geqslant 0$ for most of the remainder of the paper.

Theorem 2 For a general Lipschitz continuous $F: \mathbb{R}^{n} \rightarrow \mathbb{R}$, define

$$
F^{+}(x)=\max (F(x), 0), \quad F^{-}(x)=\min (F(x), 0)
$$

together with the open sets $\Omega^{+}=F^{-1}((0, \infty))$ and $\Omega^{-}=F^{-1}((-\infty, 0))$. Let $\phi^{ \pm}$be the solutions of (1) for $F^{ \pm}$with initial data $\phi_{0}$. Then

$$
\phi(x, t)=\left\{\begin{aligned}
\phi^{+}(x, t) & \text { for } x \in \Omega^{+} \\
\phi^{-}(x, t) & \text { for } x \in \Omega^{-} \\
\phi_{0}(x) & \text { if } F(x)=0
\end{aligned}\right.
$$

solves (1) for $F$ and with initial data $\phi_{0}$.

Furthermore, $\phi^{+} \leqslant \phi_{0} \leqslant \phi^{-}$throughout $\mathbb{R}^{n} \times[0, \infty)$, and $\phi^{ \pm}(x, t)=\phi_{0}(x)$ for all $x \notin \Omega^{ \pm}$ and $t \geqslant 0$.

Proof. The relation $\phi^{+} \leqslant \phi_{0} \leqslant \phi^{-}$follows immediately from Lemma 2 since $F^{-} \leqslant 0 \leqslant F^{+}$and $\phi_{0}$ is the solution for $F=0$ by Lemma 1. It is clear that $\phi$, as defined in (3), satisfies the initial condition $\phi(x, 0)=\phi_{0}(x)$, since this condition is imposed on both of $\phi^{ \pm}$.

The next step is to show $\phi^{+}(x, t)=\phi_{0}(x)$ for all $x \notin \Omega^{+}$and $t>0$. For this, define

$$
\tilde{\phi}(x, t)=\left\{\begin{array}{rr}
\phi^{+}(x, t) & \text { for } x \in \Omega^{+}, \\
\phi_{0}(x) & \text { if } F(x) \leqslant 0 .
\end{array}\right.
$$

Take note that $\phi^{+} \leqslant \tilde{\phi} \leqslant \phi_{0}$ since $\phi^{+} \leqslant \phi_{0}$. Because $\Omega^{+}=F^{-1}((0, \infty))$ is open, it follows that $\tilde{\phi}$ is upper semi-continuous. To see this, let $(x, t) \in \mathbb{R}^{n} \times[0, \infty)$ be arbitrary and $\left(x_{k}, t_{k}\right) \rightarrow(x, t)$. If $x \in \Omega^{+}$, then $x_{k} \in \Omega^{+}$if $k$ is large enough. Consequently,

$$
\limsup _{k \rightarrow \infty} \tilde{\phi}\left(x_{k}, t_{k}\right)=\limsup _{k \rightarrow \infty} \phi^{+}\left(x_{k}, t_{k}\right)=\phi^{+}(x, t)=\tilde{\phi}(x, t)
$$


by continuity of $\phi^{+}$. If, on the other hand, $x \notin \Omega^{+}$, then

$$
\limsup _{k \rightarrow \infty} \tilde{\phi}\left(x_{k}, t_{k}\right) \leqslant \limsup _{k \rightarrow \infty} \phi_{0}\left(x_{k}, t_{k}\right)=\phi_{0}(x, t)=\tilde{\phi}(x, t)
$$

since $\tilde{\phi} \leqslant \phi_{0}$ is always the case and $\phi_{0}$ is continuous.

We proceed to show that $\tilde{\phi}$ is a subsolution of (1) with speed $F^{+}$, which will then imply $\tilde{\phi} \leqslant \phi^{+}$

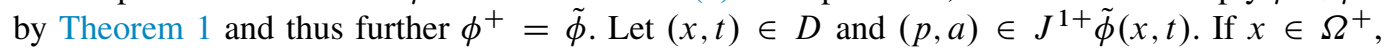
then note that $\tilde{\phi}=\phi^{+}$in a neighbourhood of $(x, t)$ since $\Omega^{+}$is open. Thus $(p, a)$ is also in $J^{1+} \phi^{+}(x, t)$, which implies $a+F^{+}(x)|p|=0 \leqslant 0$ since $\phi^{+}$is the solution for $F^{+}$. Assume now $x \in \mathbb{R}^{n} \backslash \Omega^{+}$, i. e., $F(x) \leqslant 0$. This implies $F^{+}(x)=0$ and also $\tilde{\phi}(x, t)=\phi_{0}(x)$ constantly in time. In this case, we can show that $a=0$ with the same argument as in the proof of Lemma 1. Hence also $a+F^{+}(x)|p|=0 \leqslant 0$, which shows that $\tilde{\phi}$ is, indeed, a subsolution of (1) with speed $F^{+}$. Similarly, one can show that $\phi^{-}(x, t)=\phi_{0}(x)$ for all $x \notin \Omega^{-}$and $t>0$.

It remains to verify that $\phi$ as defined in (3) is actually a solution of (1) with speed $F$. Take note that the considerations above imply that $\phi^{+}(x, t)=\phi^{-}(x, t)=\phi_{0}(x)$ whenever $F(x)=0$. Thus, $\phi$ is continuous since $\phi^{ \pm}$as well as $\phi_{0}$ are continuous. With the same argument that was used above for $\tilde{\phi}$, one can now also show that $\phi$ itself is both a sub- and supersolution of (1).

\section{Generalised distances}

As a preparation for the following results, in this section we will investigate the Eikonal equation

$$
F(x)\left|\nabla d_{y}(x)\right|=1, \quad d_{y}(y)=0
$$

for some fixed "source" $y \in \mathbb{R}^{n}$. For this stationary equation, viscosity solutions can be defined in a similar way to Definition 2. As discussed above, we assume that $F: \mathbb{R}^{n} \rightarrow[0, \infty)$ has compact support and is Lipschitz continuous with constant $L_{F}$. Consequently, it attains a maximal value, so that we can find $\bar{F} \in \mathbb{R}$ with $0 \leqslant F(x) \leqslant \bar{F}$ for all $x \in \mathbb{R}^{n}$. (Note that we always consider $F \geqslant 0$ in this section.) Dropping the requirement that $F$ has compact support for the moment and assuming $F=1$ on the whole space, one can show that $d_{y}(x)=|x-y|$ solves (4). In this simplified case, $d_{y}$ is just the usual geometric distance to $y$. (See Section 3.1 of [27] for a thorough discussion of this case.) For more general $F$, the solution $d_{y}(x)$ of (4) yields a generalised distance instead. As we will see in Theorem 3, this distance corresponds to the shortest travel time from the source $y$ to some arbitrary target $x$, with $F$ defining the allowed speed of movement at each point in space. There exists a vast literature about (4), mainly for the case that $F$ is uniformly bounded away from zero. See, for instance, Theorem 5.3 on page 132 of [29]. We will, however, not use this assumption here and deduce some relevant results for the more general case instead. As in the preceding section, let us define $\Omega^{+}=F^{-1}((0, \infty))$ as the set where $F$ is strictly positive. $\Omega^{+}$is clearly open and bounded, and each connected component of $\Omega^{+}$is also open and thus even path-connected. This, in particular, implies that the set $X_{\text {ad }}(x, y)$ defined below is not empty.

DEFINITION 3 Let $C \subset \Omega^{+}$be a connected component and $x, y \in C$. A path connecting $x$ and $y$ is a function $\xi \in W^{1, \infty}\left([0,1], \mathbb{R}^{n}\right)$ with $\xi(0)=x, \xi(1)=y$ and $\xi(t) \in C$ for all $t \in[0,1]$. We write $X_{\text {ad }}(x, y)$ for the set of all such paths from $x$ to $y$.

For $\xi \in X_{\mathrm{ad}}(x, y)$, the Euclidean arc length is defined as

$$
|\xi|=\int_{0}^{1}\left|\xi^{\prime}(t)\right| d t
$$


in the usual way. We can also define the corresponding $F$-induced length of $\xi$ as

$$
l(\xi)=\int_{0}^{1} \frac{\left|\xi^{\prime}(t)\right|}{F(\xi(t))} d t
$$

By the Sobolev embedding theorem (see, for instance, Theorem 6 on page 270 of [16]), each path $\xi \in X_{\text {ad }}(x, y)$ is continuous. Furthermore, by the continuity of $F$ also $F \circ \xi$ is continuous, and thus this function attains a minimum on the compact interval $[0,1]$. Since $\xi$ maps into $C$, this minimum must actually be strictly positive. Thus, (5) is well-defined with $0 \leqslant l(\xi)<\infty$. Also note that using the fundamental theorem of calculus, one can easily show that the straight line

$$
S_{x y}(t)=x+t(y-x)
$$

between $x$ and $y$ has the shortest possible Euclidean arc length: Let $\xi \in X_{\text {ad }}(x, y)$, then

$$
|\xi|=\int_{0}^{1}\left|\xi^{\prime}(t)\right| d t \geqslant\left|\int_{0}^{1} \xi^{\prime}(t) d t\right|=|\xi(1)-\xi(0)|=|x-y|=\left|S_{x y}\right| .
$$

Another key observation is that one can always reparametrise a given path $\xi$ such that $\left|\xi^{\prime}(t)\right|=$ $|\xi|$ is constant for all $t \in[0,1]$. This corresponds to a parametrisation by path length followed by a rescaling of the time interval from $[0,|\xi|]$ back to $[0,1]$. It is easy to see that this operation does not change any geometrical properties and leaves, in particular, $|\xi|$ and $l(\xi)$ invariant. In the following, we will most of the time assume, without loss of generality, that this is done for the considered paths. With this assumption, the path length (5) becomes

$$
l(\xi)=\int_{0}^{1} \frac{|\xi|}{F(\xi(t))} d t .
$$

Finally, note that paths $\xi \in W^{1, \infty}\left([0,1], \mathbb{R}^{n}\right)$ are Lipschitz continuous. We denote the optimal Lipschitz constant by

$$
\operatorname{Lip}(\xi)=\sup _{t \neq s \in[0,1]}\left|\frac{\xi(t)-\xi(s)}{t-s}\right| .
$$

For paths reparametrised by arc length in the way described above, it follows easily that $\operatorname{Lip}(\xi)=$ $|\xi|$.

DEFinition 4 For $x \in \mathbb{R}^{n}$, we set $d(x, x)=0$. For $y \neq x$, if there exists a connected component $C \subset \Omega^{+}$with $x, y \in C$, we define

$$
d(x, y)=\inf _{\xi \in X_{\text {ad }}(x, y)} l(\xi) .
$$

Otherwise, we set $d(x, y)=\infty$.

This defines a generalised distance $d(x, y) \in[0, \infty]$ between any two points $x$ and $y$. This distance corresponds to the "shortest travel time" between the points under the speed field $F$. If the points are not in the same connected component of $\Omega^{+}$, then each path between them must necessarily pass through intermediate points $z$ with $F(z)=0$, which justifies the definition of $d(x, y)=\infty$ in this case. This will be further clarified by Lemma 5 below. 
Since the difference between our situation and the one handled commonly in the literature is that we allow $F(x) \rightarrow 0$ as $x \rightarrow \partial \Omega^{+}$without a strictly positive lower bound, we have to pay special attention to this case. It turns out, however, that Lipschitz continuity of $F$ ensures that paths with finite length can never actually reach $\partial \Omega^{+}$:

Lemma 5 Let $C \subset \Omega^{+}$be a connected component, $X \subset C$ be compact, and choose $M>0$. Then there exists $\underline{F}>0$ such that for all $x \in X, y \in C$ and $\xi \in X_{\mathrm{ad}}(x, y)$, the condition $F(\xi(t)) \leqslant \underline{F}$ for some $t \in[0,1]$ necessarily implies $l(\xi) \geqslant M$.

Proof. Let $\xi \in X_{\text {ad }}(x, y)$ and assume that $F\left(\xi\left(t_{0}\right)\right) \leqslant \underline{F}$ for some $\underline{F}>0$ and $t_{0} \in[0,1]$. From Lipschitz continuity, we get

$$
F(\xi(t)) \leqslant \underline{F}+|\xi| L_{F}\left|t-t_{0}\right|
$$

But this also implies

$$
\frac{|\xi|}{F(\xi(t))} \geqslant \frac{|\xi|}{\underline{F}+|\xi| L_{F}\left(t_{0}-t\right)}
$$

for all $t \in\left[0, t_{0}\right]$. Hence

$$
l(\xi) \geqslant \int_{0}^{t_{0}} \frac{|\xi|}{F(\xi(t))} d t \geqslant|\xi| \int_{0}^{t_{0}} \frac{1}{\underline{F}+|\xi| L_{F}\left(t_{0}-t\right)} d t=\frac{\log \left(|\xi| L_{F} t_{0}+\underline{F}\right)-\log (\underline{F})}{L_{F}} .
$$

Now assume $F(z) \geqslant F_{0}>0$ for all $z \in X$, which is possible by compactness of $X$, and pick $x \in X$. Again by Lipschitz continuity, we have

$$
F_{0} \leqslant F(x)=F(\xi(0)) \leqslant \underline{F}+|\xi| L_{F} t_{0} \Rightarrow|\xi| L_{F} t_{0} \geqslant F_{0}-\underline{F} \geqslant \frac{F_{0}}{2}
$$

if only $F$ is chosen at most $F_{0} / 2$. In particular, $|\xi| L_{F} t_{0}$ is bounded away from zero with a constant depending only on $X$. But this gives further

$$
l(\xi) \geqslant \frac{\log \left(|\xi| L_{F} t_{0}\right)-\log (\underline{F})}{L_{F}} \geqslant \frac{\log \left(F_{0} / 2\right)-\log (\underline{F})}{L_{F}},
$$

which can be made arbitrarily large by choosing $F$ small enough. The proof is now finished if we note that the choice of $\underline{F}$ for the claim to be satisfied only depends on $X$ and $F$ but not the particular $x, y$ or $\xi$ under consideration.

Lemma 5 can be interpreted as a coercivity result: Since path lengths become infinite when approaching the boundary of $\Omega^{+}$, such paths can never be relevant for the determination of shortest paths and, consequently, the distance $d(\cdot, \cdot)$. This allows us to restrict ourselves to compact subsets of $\Omega^{+}$in these situations. As a first application of this feature, let us show the existence of a path with minimal length if $x$ and $y$ are in the same connected component (i. e., $d(x, y)<\infty)$. For this, we make use of the theorem of Arzelà-Ascoli (see Appendix C.7 in [16]) to get compactness, and use Lipschitz estimates to show lower semi-continuity. To be precise:

Lemma 6 Let $x, y \in \mathbb{R}^{n}$ with $d(x, y)<\infty$. Let $\xi \in X_{\mathrm{ad}}(x, y),\left(\xi_{k}\right) \subset X_{\mathrm{ad}}(x, y)$ and $L>0$ be a uniform Lipschitz constant for all $\xi_{k}$. Assume $\xi_{k} \rightarrow \xi$ uniformly and that all $\xi_{k}$ are parametrised by arc length. (We do not assume this to be true for $\xi$.) Then also $\xi$ is Lipschitz continuous and we have

$$
|\xi|=\int_{0}^{1}\left|\xi^{\prime}(t)\right| d t \leqslant \operatorname{Lip}(\xi) \leqslant \liminf _{k \rightarrow \infty} \operatorname{Lip}\left(\xi_{k}\right) \leqslant L
$$


Proof. For simplicity, assume that $\lim _{k \rightarrow \infty} \operatorname{Lip}\left(\xi_{k}\right)$ exists. (If that is not the case, choose a subsequence that converges to the limit inferior.) Fix $t \neq s$ and note that

$$
\left|\frac{\xi(t)-\xi(s)}{t-s}\right|=\lim _{k \rightarrow \infty}\left|\frac{\xi_{k}(t)-\xi_{k}(s)}{t-s}\right| \leqslant \lim _{k \rightarrow \infty} \operatorname{Lip}\left(\xi_{k}\right) .
$$

Since this is true for arbitrary $t$ and $s$, it also holds in the supremum to give $\operatorname{Lip}(\xi) \leqslant$ $\lim _{k \rightarrow \infty} \operatorname{Lip}\left(\xi_{k}\right)$. The remaining estimates follow immediately.

Lemma 7 Let $x, y \in \mathbb{R}^{n}$ and $d(x, y)<\infty$. Then there exists $\xi \in X_{\mathrm{ad}}(x, y)$ such that $d(x, y)=$ $l(\xi)$.

Proof. By definition of $d(x, y)$, we can find a minimising sequence $\left(\xi_{k}\right)$ with $l\left(\xi_{k}\right) \rightarrow d(x, y)$. Assume that each $\xi_{k}$ is parametrised by arc length. This implies that there exists a uniform Lipschitz constant $L$ for all $\xi_{k}$, since each has Lipschitz constant Lip $\left(\xi_{k}\right)=\left|\xi_{k}\right|$. The arc lengths $\left|\xi_{k}\right|$, in turn, are bounded uniformly because the sequence minimises $l\left(\xi_{k}\right)$ and

$$
\frac{\operatorname{Lip}\left(\xi_{k}\right)}{\bar{F}} \leqslant \int_{0}^{1} \frac{\left|\xi_{k}\right|}{F\left(\xi_{k}(t)\right)} d t=l\left(\xi_{k}\right) .
$$

By the theorem of Arzelà-Ascoli, there exists a continuous path $\xi$ that is the uniform limit of a subsequence of $\left(\xi_{k}\right)$. Without loss of generality, assume that the subsequence is $\left(\xi_{k}\right)$ itself, so that $\xi_{k} \rightarrow \xi$ uniformly. Furthermore, also $\xi$ is Lipschitz continuous with Lipschitz constant $L$ by Lemma 6. Thus $\xi \in X_{\text {ad }}(x, y)$. Note that, in particular, the image of $\xi$ has to lie inside of $C \subset \Omega^{+}$. If this were not the case, then Lemma 5 would imply that the sequence $\left(\xi_{k}\right)$ has unbounded path lengths. This would contradict the assumption that it is a minimising sequence. By definition of $d(x, y)$, it is clear that $l(\xi) \geqslant d(x, y)$. It remains to show that also $l(\xi) \leqslant d(x, y)=$ $\lim _{k \rightarrow \infty} l\left(\xi_{k}\right)$ holds.

For this, define $g(\tau)=1 / F(\xi(\tau))$. Since the image of $\xi$ lies inside of $\Omega^{+}$, we know that $F \circ \xi$ is bounded away from zero. Hence, $g$ is Lipschitz continuous with some constant $L_{g}$. Assume that we have some partition $\ell$ of $[0,1]$ into intervals $I_{i}=\left[t_{i}, t_{i+1}\right]$ and that $\ell$ has fineness $h=\sup _{i}\left(t_{i+1}-\right.$ $\left.t_{i}\right)$. Choose $\epsilon>0$ arbitrary and pick $K \in \mathbb{N}$ such that $\operatorname{Lip}\left(\xi ; I_{i}\right) \leqslant \operatorname{Lip}\left(\xi_{k} ; I_{i}\right)+\epsilon$ for all intervals $I_{i} \in \mathcal{l}$ and $k \geqslant K$. This is possible due to Lemma 6 (applied to the intervals $I_{i}$ instead of $[0,1]$ ). Finally, since $\xi_{k} \rightarrow \xi$ uniformly, we also have the uniform convergence $g_{k}=1 /\left(F \circ \xi_{k}\right) \rightarrow g$. Hence, we may assume $g(\tau) \leqslant g_{k}(\tau)+\epsilon$ for all $\tau \in[0,1]$ and $k \geqslant K$.

Now, using again the estimates in Lemma 6 , we get:

$$
\begin{aligned}
\int_{0}^{1} g(\tau)\left|\xi^{\prime}(\tau)\right| d \tau & \leqslant \sum_{I_{i} \in \mathcal{l}} \sup _{\tau \in I_{i}} g(\tau) \cdot\left(t_{i+1}-t_{i}\right) \cdot \operatorname{Lip}\left(\xi ; I_{i}\right) \\
& \leqslant \sum_{I_{i} \in \mathcal{l}} \sup _{\tau \in I_{i}} g(\tau) \cdot\left(\int_{I_{i}}\left|\xi_{k}^{\prime}(\tau)\right| d \tau+\left(t_{i+1}-t_{i}\right) \epsilon\right)
\end{aligned}
$$

Since $g$ is Lipschitz continuous, we also know that

$$
\sup _{\tau \in I_{i}} g(\tau) \leqslant \inf _{\tau \in I_{i}} g(\tau)+h L_{g} .
$$


Furthermore, clearly

$$
\inf _{\tau \in I_{i}} g(\tau) \int_{I_{i}}\left|\xi_{k}^{\prime}(\tau)\right| d \tau \leqslant \int_{I_{i}} g(\tau)\left|\xi_{k}^{\prime}(\tau)\right| d \tau \leqslant \int_{I_{i}}\left(g_{k}(\tau)+\epsilon\right)\left|\xi_{k}^{\prime}(\tau)\right| d \tau
$$

All that taken together yields

$$
\begin{aligned}
\int_{0}^{1} g(\tau) \mid & \xi^{\prime}(\tau) \mid d \tau \\
& \leqslant \sum_{I_{i} \in \ell}\left(\int_{I_{i}}(g(\tau)+\epsilon)\left|\xi_{k}^{\prime}(\tau)\right| d \tau+h L_{g} \int_{I_{i}}\left|\xi_{k}^{\prime}(\tau)\right| d \tau+\left(t_{i+1}-t_{i}\right) \epsilon\|g\|_{\infty}\right) \\
& =\int_{0}^{1} g_{k}(\tau)\left|\xi_{k}^{\prime}(\tau)\right| d \tau+\left(\epsilon+h L_{g}\right)\left|\xi_{k}\right|+\epsilon\|g\|_{\infty} .
\end{aligned}
$$

Recall that $\epsilon$ and $\ell$ were arbitrary. Thus, also $h$ can be made small. Note that $\left|\xi_{k}\right|$ is bounded for $k \rightarrow \infty$. This now implies the claim, since

$$
l(\xi)=\int_{0}^{1} g(\tau)\left|\xi^{\prime}(\tau)\right| d \tau \leqslant \liminf _{k \rightarrow \infty} \int_{0}^{1} g_{k}(\tau)\left|\xi_{k}^{\prime}(\tau)\right| d \tau=\lim _{k \rightarrow \infty} l\left(\xi_{k}\right)=d(x, y) .
$$

We continue by deriving some fundamental properties of and estimates for the path lengths and the distance function $d(\cdot, \cdot)$ :

Lemma 8 Let $x, y \in \mathbb{R}^{n}$ be arbitrary. Then

$$
|x-y| \leqslant \bar{F} \cdot d(x, y) \text {. }
$$

Proof. For $d(x, y)=\infty$ and for $x=y$, the claim is obvious. Thus assume that $x$ and $y$ are in the same connected component of $\Omega^{+}$, and let $\xi \in X_{\text {ad }}(x, y)$. Then

$$
l(\xi)=\int_{0}^{1} \frac{|\xi|}{F(\xi(x))} d t \geqslant \frac{|\xi|}{\bar{F}} \geqslant \frac{|x-y|}{\bar{F}}
$$

where we have used the assumption of a reparametrised $\xi$ and (6).

This result gives an important estimate relating $d(x, y)$ and $|x-y|$. If we use Lipschitz continuity of $F$ in addition, also more precise estimates are possible especially for points close to each other. In particular, we get the following localised version of Lemma 8:

Lemma 9 Let $x \in \Omega^{+}$and $y \in \mathbb{R}^{n}$. Then we have:

$$
|x-y| \leqslant \frac{F(x)}{L_{F}}\left(e^{L_{F} d(x, y)}-1\right)
$$

If furthermore $L_{F}|x-y|<F(x)$, then also

$$
\begin{aligned}
d(x, y) \leqslant l\left(S_{x y}\right) & \leqslant \frac{1}{L_{F}} \log \frac{F(x)}{F(x)-L_{F}|x-y|}, \\
|x-y| & \geqslant \frac{F(x)}{L_{F}}\left(1-e^{-L_{F} d(x, y)}\right) .
\end{aligned}
$$

As before, $S_{x y} \in X_{\mathrm{ad}}(x, y)$ denotes the straight line between $x$ and $y$. 
Proof. If $d(x, y)=\infty$, the first claim is clear. For the remaining case, we choose $\xi \in X_{\mathrm{ad}}(x, y)$ arbitrary. Let $\xi$ be parametrised by its arc length $|\xi|$. Then $|\xi| \geqslant|x-y|$ and Lipschitz continuity of $F$ implies

$$
F(\xi(t)) \leqslant F(x)+L_{F}|\xi(t)-x|=F(x)+L_{F}\left|\int_{0}^{t} \xi^{\prime}(\tau) d \tau\right| \leqslant F(x)+L_{F} t|\xi|
$$

for all $t \in[0,1]$. This yields

$$
\begin{aligned}
l(\xi) & =\int_{0}^{1} \frac{|\xi|}{F(\xi(t))} d t \geqslant \int_{0}^{1} \frac{|\xi|}{F(x)+L_{F} t|\xi|} d t \\
& =\frac{1}{L_{F}} \log \frac{F(x)+|\xi| L_{F}}{F(x)} \geqslant \frac{1}{L_{F}} \log \left(1+\frac{|x-y| L_{F}}{F(x)}\right),
\end{aligned}
$$

which also holds in the infimum over all possible paths $\xi$. Thus

$$
e^{L_{F} d(x, y)} \geqslant 1+\frac{|x-y| L_{F}}{F(x)},
$$

which further implies the first estimate.

For the second estimate, note that $d(x, y) \leqslant l\left(S_{x y}\right)$ as well as $\left|S_{x y}\right|=|x-y|$. Lipschitz continuity tells us that

$$
F\left(S_{x y}(t)\right) \geqslant F(x)-L_{F}\left|S_{x y}(t)-x\right|=F(x)-L_{F} t|x-y|,
$$

and by our assumption this expression is guaranteed to be positive for all $t \in[0,1]$. This implies also, in particular, that $S_{x y}$ lies entirely inside of $\Omega^{+}$. Thus we find

$$
\begin{aligned}
d(x, y) \leqslant l\left(S_{x y}\right) & =\int_{0}^{1} \frac{\left|S_{x y}\right|}{F\left(S_{x y}(t)\right)} d t \leqslant \int_{0}^{1} \frac{|x-y|}{F(x)-L_{F} t|x-y|} d t \\
& =\frac{1}{L_{F}} \log \frac{F(x)}{F(x)-L_{F}|x-y|} .
\end{aligned}
$$

The third estimate is just an equivalent reformulation of the second one.

The following is a variant of Theorem 5.1 on page 117 of [29], adapted for our problem (4):

Lemma $10 d(\cdot, \cdot)$ defines a metric on each connected component of $\Omega^{+}$.

Proof. By Definition 4, $d(x, x)=0$ for each $x \in \mathbb{R}^{n}$. Let $C \subset \Omega^{+}$be a connected component, $x, y \in C$ and $x \neq y$. For each $\xi \in X_{\text {ad }}(x, y)$, we can define $\xi_{c}(t)=\xi(1-t)$, which yields $\xi_{c} \in X_{\text {ad }}(y, x)$ with $l(\xi)=l\left(\xi_{c}\right)$. Hence $d(x, y)=d(y, x)$ holds also in this case. Let now $z \in C$ be given in addition. We use Lemma 7 to choose $\xi_{1} \in X_{\text {ad }}(x, z)$ and $\xi_{2} \in X_{\text {ad }}(z, y)$ with

$$
l\left(\xi_{1}\right)+l\left(\xi_{2}\right)=d(x, z)+d(z, y) .
$$

Let us define $\xi$ as the concatenation of $\xi_{1}$ and $\xi_{2}$ with subsequent reparametrisation. Consequently, $\xi \in X_{\text {ad }}(x, y)$ and

$$
d(x, y) \leqslant l(\xi)=l\left(\xi_{1}\right)+l\left(\xi_{1}\right)=d(x, z)+d(z, y) .
$$


It remains to verify that we also have non-degeneracy in the form of

$$
d(x, y)=0 \Rightarrow x=y .
$$

This, however, follows directly from Lemma 8.

Lemma 11 Let $X \subset \Omega^{+}$be compact and convex. Then

$$
d(x, y) \leqslant L|x-y|
$$

for all $x, y \in X$, where $L=1 / \underline{F}$ and $\underline{F}>0$ is the minimum of $F$ over $X$.

Proof. Let $F>0$ be the minimum of $F$ on the compact set $X$ and choose $x, y \in X$. We consider the straight line $S_{x y}$ as particular path in $X_{\text {ad }}(x, y)$. Convexity ensures that $S_{x y}(t) \in X$ for all $t \in[0,1]$. The claim now follows with

$$
d(x, y) \leqslant l\left(S_{x y}\right)=\int_{0}^{1} \frac{\left|S_{x y}\right|}{F\left(S_{x y}(t)\right)} d t \leqslant \frac{|x-y|}{\underline{F}} .
$$

Lemma $12 d(\cdot, \cdot)$ is continuous in both arguments on each connected component of $\Omega^{+}$.

Proof. Let $C \subset \Omega^{+}$be a connected component and $x \in C$. By symmetry according to Lemma 10 it is sufficient to show that $d_{x}(\cdot)=d(\cdot, x)$ is continuous on $C$. First, we show that $d_{x}$ is continuous at $x$ itself. For this, let $\left(x_{k}\right) \subset C$ with $x_{k} \rightarrow x$ as $k \rightarrow \infty$ and pick $\delta>0$ such that $\overline{B_{\delta}(x)} \subset C$. This is possible since $C$ is open. Assume for simplicity and without loss of generality that $\left|x_{k}-x\right|<\delta$ for all $k \in \mathbb{N}$. Since $\overline{B_{\delta}(x)}$ is compact and convex, we can apply Lemma 11 in order to deduce

$$
0 \leqslant d_{x}\left(x_{k}\right)=d\left(x_{k}, x\right) \leqslant L\left|x_{k}-x\right|
$$

for some constant $L$. Since the right-hand side vanishes with $x_{k} \rightarrow x$, we find that also the limit $d_{x}\left(x_{k}\right) \rightarrow 0=d_{x}(x)$ must hold. This shows continuity of $d_{x}$ at $x$.

Now let $x, y \in C$ be arbitrary. We will show that $d_{y}=d(\cdot, y)$ is continuous at $x$. For this, choose $\epsilon>0$. From the previous argument, we know that there exists $\delta>0$ such that $d\left(x, x^{\prime}\right)<$ $\epsilon$ if only $\left|x^{\prime}-x\right|<\delta$. Applying the triangle inequality and the reverse triangle inequality from Lemma 10 for some intermediate point $x^{\prime} \in B_{\delta}(x)$, we get

$$
d(x, y) \leqslant d\left(x, x^{\prime}\right)+d\left(x^{\prime}, y\right) \Rightarrow d\left(x^{\prime}, y\right) \geqslant d(x, y)-d\left(x, x^{\prime}\right) \geqslant d(x, y)-\epsilon
$$

and

$$
d\left(x^{\prime}, y\right) \leqslant d\left(x^{\prime}, x\right)+d(x, y) \leqslant d(x, y)+\epsilon .
$$

Hence we find $\left|d\left(x^{\prime}, y\right)-d(x, y)\right|<\epsilon$ whenever $\left|x^{\prime}-x\right|<\delta$, which is the claimed continuity.

After this quite general discussion, let us get back to the task of solving (4). For the case of a strictly positive, uniform lower bound $F \geqslant \underline{F}>0$, this is well-understood. In order to reduce our more general problem to the known results, we will make use of Lemma 5. As a first step, let us introduce a sequence of cut-off speeds: 
Definition 5 For given $\underline{F}>0$, we define $\tilde{F}(x)=\max (F(x), F)$. The notation $l_{F}(\xi)$ and $d_{\underline{F}}(x, y)$ will be used for path lengths and distances according to Definition 3 and Definition 4 , respectively, based on $\tilde{F}$ instead of the original $F$.

Note that $\tilde{F}$ has no longer compact support, but this is no problem since $\tilde{F}$ is still bounded as long as the original speed field $F$ is bounded. This guarantees that all arguments go through nevertheless. The distance $d_{F}(x, y)$ is equivalent to $L(x, y)$ given in (47) on page 116 of [29] when using $n(x)=1 / \tilde{F}(x)$. Theorem 5.1 on page 117 of [29] will be the main tool on which we build our results. There, it is shown that $d_{\underline{F}}(\cdot, y)$ is a viscosity solution of (4) if the speed $F$ is replaced by $\tilde{F}$. We will now work on reducing our situation to the case where the result of [29] is applicable.

Lemma 13 Let $C \subset \Omega^{+}$be a connected component and $X \subset C$ be compact. Then there exists $\underline{F}>0$ such that $d(x, y)=d_{F^{\prime}}(x, y)$ for all $\underline{F}^{\prime} \in(0, \underline{F}]$ and $x, y \in X$.

Proof. Let $\tilde{F}$ be the cut-off speed for some $\underline{F}>0$. Then clearly $F(x) \leqslant \tilde{F}(x)$, which implies $l_{\underline{F}}(\xi) \leqslant l(\xi)$ for every path $\xi$. Hence also $d_{\underline{F}}(x, y) \leqslant d(x, y)$. It remains to show that our assumptions actually imply equality. Furthermore, if we show the result for a single $F$ as cut-off threshold, it must also hold for all smaller thresholds. This is the case because $\underline{F}^{\prime} \leqslant \underline{F}$ implies $d(x, y)=d_{\underline{F}}(x, y) \leqslant d_{\underline{F}^{\prime}}(x, y) \leqslant d(x, y)$. Thus, it remains to show that $d(x, y) \leqslant d_{\underline{F}}(x, y)$ holds for some threshold $\bar{F}>0$.

Set $M=\max _{x, y \in X} \bar{d}(x, y)$, which is well-defined and finite because of Lemma 12 and since $X$ is compact. We now apply Lemma 5 for this $M$ to get a corresponding positive threshold $\underline{F}$. We can assure $F(x)>\underline{F}>0$ for all $x \in X$ by decreasing $\underline{F}$ further as necessary. Now, assume that $d_{F}(x, y)<d(x, y)$ for some $x, y \in X$. Choose a minimising path $\xi \in X_{\mathrm{ad}}(x, y)$ with $l_{\underline{F}}(\xi)=d_{\underline{F}}(x, y)$. Consequently,

$$
l_{\underline{F}}(\xi)<d(x, y) \leqslant l(\xi) .
$$

This, however, implies that there exists $t \in[0,1]$ with $F(\xi(t))<\underline{F}$. If that would not be the case, then $F(\xi(t))=\tilde{F}(\xi(t))$ for all $t \in[0,1]$ and thus the lengths would have to coincide. Define now

$$
t_{0}=\inf \{t \in[0,1] \mid F(\xi(t)) \leqslant \underline{F}\}=\min \{t \in[0,1] \mid F(\xi(t)) \leqslant \underline{F}\},
$$

which is well-defined because the set is non-empty and $F \circ \xi$ is continuous. Furthermore, for all $t \in\left[0, t_{0}\right]$ we have $F(\xi(t)) \geqslant \underline{F}$ and consequently $F(\xi(t))=\tilde{F}(\xi(t))$. Also, $t_{0}>0$ because $x \in X$ and thus $F(\xi(0))=F(x)>\underline{F}$. Now define a new path $\xi_{1}(t)=\xi\left(t / t_{0}\right)$. We have $\xi_{1} \in$ $X_{\text {ad }}\left(x, \xi\left(t_{0}\right)\right)$, and since $F$ equals $\tilde{F}$ along the path, also $l\left(\xi_{1}\right)=l_{\underline{F}}\left(\xi_{1}\right)$. Since $F\left(\xi_{1}(1)\right)=\underline{F}$, we can apply Lemma 5 now to $\xi_{1}$ to deduce $l\left(\xi_{1}\right) \geqslant M$. This is a contradiction, since

$$
l_{\underline{F}}(\xi) \geqslant l_{\underline{F}}\left(\xi_{1}\right)=l\left(\xi_{1}\right) \geqslant M
$$

and we know $l_{\underline{F}}(\xi)<d(x, y) \leqslant M$.

Theorem 3 Let $C \subset \Omega^{+}$be a connected component and $y \in C$ be fixed. Then $d_{y}(\cdot)=d(\cdot, y)$ is a viscosity solution of (4) in $C \backslash\{y\}$.

Proof. Note that $d_{y}$ is continuous on $C$ by Lemma 12, and that the boundary condition is trivially satisfied because of $d_{y}(y)=d(y, y)=0$. It remains to show that $d_{y}$ satisfies $F(x)\left|\nabla d_{y}(x)\right|=1$ in the viscosity sense. For this, let $x \in C \backslash\{y\}$ be fixed and $p \in J^{1+} d_{y}(x)$. Now choose 
$Y \subset C$ compact, connected, with smooth boundary and such that $x, y \in Y^{\circ}$. Choose $\underline{F}$ for $Y$ according to Lemma 13 and such that $\underline{F} \leqslant \min _{z \in Y} F(z)$ in addition. Then $\tilde{F}=F$ on $Y$ and $d_{y}\left(x^{\prime}\right)=d\left(x^{\prime}, y\right)=d_{\underline{F}}\left(x^{\prime}, y\right)$ for all $x^{\prime} \in Y$. The latter property holds, in particular, also in a neighbourhood of $x$ so that $p \in J^{1+} d_{F}(x, y)$ must be true. Since $d_{F}(\cdot, y)$ solves (4) with $\tilde{F}$ in $Y^{\circ} \backslash\{y\}$ in the viscosity sense as noted above according to Theorem 5.1 on page 117 of [29], this implies $F(x)|p|=\tilde{F}(x)|p| \leqslant 0$. This, however, is all we need to show that $d_{y}$ is a viscosity subsolution of (4) on the whole of $C \backslash\{y\}$. By a symmetric argument one can also show that it is a viscosity supersolution.

We conclude this section with a final auxiliary result that will be useful later:

Lemma 14 Let $X \subset \mathbb{R}^{n}$ be closed, $x \in X$ and assume that $d(x, y) \geqslant t$ for some $t \geqslant 0$ and all $y \in \partial X$. Then $d(x, y)>t$ for all $y \notin X$. More precisely: If $y \notin X$ and $\delta>0$ are such that $B_{\delta}(y) \cap X=\emptyset$, then $d(x, y) \geqslant t+\delta / \bar{F}$.

Proof. Let $y \notin X$, then there exists $\delta>0$ such that $B_{\delta}(y) \subset \mathbb{R}^{n} \backslash X$ since $\mathbb{R}^{n} \backslash X$ is open. Consequently, $\left|x^{\prime}-y\right| \geqslant \delta>0$ for all $x^{\prime} \in X$. Clearly, $y \neq x$ and if $x \notin \Omega^{+}, y \notin \Omega^{+}$or they are not in the same connected component of $\Omega^{+}$, then $d(x, y)=\infty>t$ holds. So assume that $x, y \in \Omega^{+}$are in the same connected component and choose $\xi \in X_{\mathrm{ad}}(x, y)$. Since $\xi$ is continuous, the set $\xi^{-1}(X) \subset[0,1]$ is closed and since it is also bounded, it is compact. Define thus

$$
t_{0}=\max \{t \in[0,1] \mid \xi(t) \in X\} \text { and } y_{0}=\xi\left(t_{0}\right) .
$$

Then $y_{0} \in X$ and furthermore $y_{0} \in \partial X$ since every sequence $\left(\xi\left(t_{k}\right)\right)$ with $t_{k} \rightarrow t_{0}^{+}$from above is in $\mathbb{R}^{n} \backslash X$ and converges to $y_{0}$. Denote the two parts of $\xi$ up to and starting at $y_{0}$ by $\xi_{1}$ and $\xi_{2}$, respectively. Then $\xi_{1} \in X_{\text {ad }}\left(x, y_{0}\right), \xi_{2} \in X_{\text {ad }}\left(y_{0}, y\right)$ and $l(\xi)=l\left(\xi_{1}\right)+l\left(\xi_{2}\right)$. Since $y_{0} \in \partial X$, we know by our assumption that $l\left(\xi_{1}\right) \geqslant d\left(x, y_{0}\right) \geqslant t$. Furthermore, Lemma 8 implies that

$$
l\left(\xi_{2}\right) \geqslant d\left(y_{0}, y\right) \geqslant \frac{\left|y_{0}-y\right|}{\bar{F}} \geqslant \frac{\delta}{\bar{F}} .
$$

Both estimates together imply $l(\xi) \geqslant t+\delta / \bar{F}$. Since $\xi$ was arbitrary, also $d(x, y) \geqslant t+\delta / \bar{F}>t$ follows by taking the infimum over all possible paths.

Note that Lemma 14 can be interpreted as a variant of the classical minimum principle: If $x \in \mathbb{R}^{n}$ is fixed and $Y \subset \mathbb{R}^{n}$ open with $x \notin Y$, then $d(x, \cdot)$ attains its minimum over $\bar{Y}$ at $\partial Y$. (The complement $\mathbb{R}^{n} \backslash Y$ takes the role of the closed set $X$ in the lemma.) A corresponding maximum principle does, however, not hold in general: If $F(y)$ is very small or even zero, then all paths connecting $x$ to $y$ may have a larger length than paths "circling around" $y$. In this case, $d(x, y)$ can, indeed, have a local maximum at $y$. See also [3], where a more general result is derived for viscosity solutions. The Eikonal equation, in particular, is covered by Example 5. A discussion of the classical minimum and maximum principles for harmonic functions can be found in Section 2.5 of [22].

\section{The Hopf-Lax formula}

We now turn our attention from the stationary problem (4) considered in Section 3 back to the timedependent level-set equation (1). As before, we assume that $F$ is Lipschitz continuous with constant 
$L_{F}$ and has compact support, and that also $\phi_{0}$ is Lipschitz continuous with constant $L_{\phi_{0}}$. Since we are particularly interested in the evolving geometries described by the zero level set of $\phi(\cdot, t)$, we also introduce the notation

$$
\Omega_{t}=\phi(\cdot, t)^{-1}((-\infty, 0)), \quad \Gamma_{t}=\phi(\cdot, t)^{-1}(\{0\}) .
$$

Clearly, $\Omega_{t}$ is open for all $t \geqslant 0$. Similarly, the set $\Gamma_{t} \cup \Omega_{t}=\phi(\cdot, t)^{-1}((-\infty, 0])$ is closed since $\phi$ is continuous. Note, though, that $\Gamma_{t}$ need not be the topological boundary $\partial \Omega_{t}$ of $\Omega_{t}$. If $\phi$ is "degenerate", $\Gamma_{t}$ may contain interior points. This effect is called fattening and will be discussed in Subsection 5.1 below.

From classical optimal-control theory, it is well-known that the level-set equation (1) can be related to a Mayer problem. For a thorough discussion, see Section III.3 of [1] or Section 3.3 of [27]. Below, we state the main arguments only briefly. For this, let us consider $F \geqslant 0$ for a moment. We define

$$
S_{t}(x)=\left\{\xi \in W^{1, \infty}\left([0, t], \mathbb{R}^{n}\right)|\xi(0)=x,| \xi^{\prime}(\tau) \mid \leqslant F(\xi(\tau)) \text { for all } \tau \in[0, t]\right\} .
$$

This is the set of paths starting in $x$ with length at most $t$. While it is similar in spirit to the set $X_{\text {ad }}(x, y)$ of Definition 3 used above, there is a slight difference: Before, we fixed both end points of the path and were interested in the path length. Now, we fix the starting point and the length, and consider possible end points. These paths can be used to define the reachable set from $x$ in time $t$ as

$$
R_{t}(x)=\left\{\xi(t) \mid \xi \in S_{t}(x)\right\} .
$$

We are now interested in the following minimisation problem:

$$
\phi(x, t)=\inf _{\xi \in S_{t}(x)} \phi_{0}(\xi(t))=\inf _{y \in R_{t}(x)} \phi_{0}(y)
$$

Standard arguments show that the value function $\phi$ of this problem is a viscosity solution of the corresponding Hamilton-Jacobi-Bellman equation. This equation, in turn, is nothing else than the level-set equation (1). Furthermore, one can also relate the reachable set to the distances discussed in the previous Section 3. This yields

$$
R_{t}(x)=\left\{y \in \mathbb{R}^{n} \mid d(x, y) \leqslant t\right\} .
$$

Note that this set is compact, so that the infimum in (8) is actually a minimum. Thus, we have established the following Hopf-Lax formula for the level-set equation:

Theorem 4 Let $F \geqslant 0$. The Hopf-Lax formula

$$
\phi(x, t)=\min \left\{\phi_{0}(y) \mid y \in \mathbb{R}^{n}, d(x, y) \leqslant t\right\}
$$

gives the viscosity solution of the level-set equation (1) in $\mathbb{R}^{n} \times[0, \infty)$.

This formula can also be derived for the case $F \geqslant \underline{F}>0$ from Theorem 3.1 on page 140 of [7]. To remove the required lower bound and show Theorem 4, one can then proceed with cut-off arguments as in the proof of Theorem 3. However, we believe that the derivation based directly on the Mayer problem is the most straight-forward argument. A similar derivation based on control theory is also given in [19], leading to Theorem 3.1 in the paper. 
Based on this representation formula (9) shown for the level-set function $\phi$, we will now proceed to derive corresponding formulas describing the evolving sets $\Omega_{t}, \Gamma_{t}$ and $\Gamma_{t} \cup \Omega_{t}$ themselves. The crucial ingredient in those formulas is the distance between a point $x$ and the initial set (not just a single point as given by $d(x, \cdot))$. This distance corresponds to the time it takes the evolving front to arrive at $x$ :

DEFINITION 6 Let $F \geqslant 0$ and denote the distance of Definition 4 by $d(\cdot, \cdot)$. For $x \in \mathbb{R}^{n}$, we set

$$
d_{0}(x)=\inf _{y \in \Gamma_{0} \cup \Omega_{0}} d(x, y), \quad d_{0}^{\prime}(x)=\inf _{y \in \Omega_{0}} d(x, y) .
$$

If $C$ is a connected component of $\Omega^{+}$, then $d_{0}$ is finite on the whole of $C$ if and only if $C$ contains a part of the initial domain $\Gamma_{0} \cup \Omega_{0}$. If this is not the case, then the distance is infinite on the whole of $C$. For $d_{0}^{\prime}$, a corresponding statement is true.

Take note that it follows immediately from Definition 6 that $d_{0}(x) \leqslant d_{0}^{\prime}(x)$ must be true for all $x \in \mathbb{R}^{n}$. We will show now that strict inequality can only hold if $\Gamma_{0}$ has non-empty interior. This is an unusual situation in applications, although we have not excluded it so far. Also note that the infimum is actually a minimum if we take it over a closed set. (The range of potential minimisers $y$ is automatically bounded since we have $|F| \leqslant \bar{F}$, which means that points too far away can never minimise the distance. This follows from Lemma 8.)

Lemma 15 Let $0 \leqslant F \leqslant \bar{F}$ and $x \in \mathbb{R}^{n}$ be arbitrary. Then there exist $y \in \Gamma_{0} \cup \Omega_{0}$ and $y^{\prime} \in \overline{\Omega_{0}}$ with $d_{0}(x)=d(x, y)$ and $d_{0}^{\prime}(x)=d\left(x, y^{\prime}\right)$.

Furthermore, if $\overline{\Omega_{0}}=\Gamma_{0} \cup \Omega_{0}$ and either $x \in \Omega^{+}$or $x \notin \Gamma_{0}$, then $d_{0}(x)=d_{0}^{\prime}(x)$.

Proof. Let $x \in \mathbb{R}^{n}$ be given. Consider the case $x \notin \Omega^{+}$first. If $x \in \Gamma_{0} \cup \Omega_{0}$, then $d_{0}(x)=$ $d(x, x)=0$ and the claim is true. If this is not the case, then $d_{0}(x)=d(x, y)=\infty$ for all $y \neq x$, and the claim also holds. The same argument can be used for $\Omega_{0}$ and $d_{0}^{\prime}$. For the second statement, we only have to consider $x \notin \Gamma_{0}$ since $x \notin \Omega^{+}$by assumption. But then either $d_{0}(x)=d_{0}^{\prime}(x)=0$ if $x \in \Omega_{0}$, or otherwise $d_{0}(x)=d_{0}^{\prime}(x)=\infty$ since then $x \notin \Gamma_{0} \cup \Omega_{0}$.

Now, assume that $x \in C$ where $C \subset \Omega^{+}$is a connected component. If $d_{0}(x)=\infty$, then also $C \cap\left(\Gamma_{0} \cup \Omega_{0}\right)=\varnothing$ and we can choose $y$ to be any element of $\Gamma_{0} \cup \Omega_{0}$. The same applies if $d_{0}^{\prime}(x)=\infty$. So assume from now on that $d_{0}(x)$ and $d_{0}^{\prime}(x)$ are both finite. This together with Lemma 5 implies that there exists a compact set $X \subset C$ such that

$$
d_{0}(x)=\inf _{y \in X \cap\left(\Gamma_{0} \cup \Omega_{0}\right)} d(x, y), \quad d_{0}^{\prime}(x)=\inf _{y \in X \cap \Omega_{0}} d(x, y) .
$$

To see this, choose $y \in C$ arbitrarily for a moment. Then $d(x, y)<\infty$. According to Lemma 5, there exists $\underline{F}>0$ such that $d(x, \tilde{y})>d(x, y)$ for all $\tilde{y}$ with $F(\tilde{y})<\underline{F}$. Thus, defining $X=$ $F^{-1}([\underline{F}, \bar{F}])$ ensures (10). If this set is not bounded, we can, furthermore, choose some radius $R>0$ such that $d(x, \tilde{y})>d(x, y)$ for all $\tilde{y}$ with $|x-\tilde{y}|>R$ based on Lemma 8. This allows us to use the compact set $X \cap \overline{B_{R}(x)}$ instead of $X$ itself.

Note that $d(x, \cdot)$ is finite and continuous when restricted to $X$. By taking a minimising sequence and using this continuity as well as compactness of the sets $X \cap\left(\Gamma_{0} \cup \Omega_{0}\right)$ and $X \cap \overline{\Omega_{0}}$, we see that the infima in (10) are actually minima. If $\overline{\Omega_{0}}=\Gamma_{0} \cup \Omega_{0}$ and $y \in \Gamma_{0} \cup \Omega_{0}$ is chosen with $d_{0}(x)=d(x, y)$, then

$$
d_{0}(x)=d(x, y) \geqslant d_{0}^{\prime}(x) \geqslant d_{0}(x),
$$

showing equality between $d_{0}(x)$ and $d_{0}^{\prime}(x)$. 
Theorem 5 Let $F \geqslant 0$. Then the evolving sets can be represented as

$$
\begin{aligned}
\Gamma_{t} \cup \Omega_{t} & =\left\{x \in \mathbb{R}^{n} \mid d_{0}(x) \leqslant t\right\}, \\
\Omega_{t} & =\left\{x \in \mathbb{R}^{n} \mid d_{0}^{\prime}(x)<t\right\}, \\
\Gamma_{t} & =\left\{x \in \mathbb{R}^{n} \mid d_{0}(x) \leqslant t \leqslant d_{0}^{\prime}(x)\right\}
\end{aligned}
$$

for all $t>0$. If $\overline{\Omega_{0}}=\Gamma_{0} \cup \Omega_{0}$, then the last relation states that

$$
\Gamma_{t}=\left\{x \in \mathbb{R}^{n} \mid d_{0}(x)=t\right\} \cup\left(\Gamma_{0} \backslash \Omega^{+}\right) .
$$

Proof. We use Theorem 4 to express $\phi$ by (9). Let $x \in \Gamma_{t} \cup \Omega_{t}$. By definition, this means $\phi(x, t) \leqslant$ 0 . Hence (9) implies that there exists $y \in \Gamma_{0} \cup \Omega_{0}$ with $d(x, y) \leqslant t$. This, in turn, yields $d_{0}(x) \leqslant$ $d(x, y) \leqslant t$. The other way round, let $d_{0}(x) \leqslant t$. By Lemma 15, there exists $y \in \Gamma_{0} \cup \Omega_{0}$ with $d(x, y)=d_{0}(x) \leqslant t$, such that $\phi(x, t) \leqslant \phi_{0}(y) \leqslant 0$ by (9) and thus $x \in \Gamma_{t} \cup \Omega_{t}$.

Now assume that $d_{0}^{\prime}(x)<t$. Applying Lemma 15 again, we find that there exists $y \in \overline{\Omega_{0}}$ with $d(x, y)=d_{0}^{\prime}(x)<t$. Thus, continuity of $d(x, \cdot)$ implies that there also exists $y^{\prime} \in \Omega_{0}$ with $d\left(x, y^{\prime}\right)<t$. Hence (9) yields $\phi(x, t) \leqslant \phi_{0}\left(y^{\prime}\right)<0$ and, consequently, $x \in \Omega_{t}$. If, on the other hand, $x \in \Omega_{t}$ and thus $\phi(x, t)<0$, there exists $y \in \Omega_{0}$ with $d(x, y) \leqslant t$. This implies at least $d_{0}^{\prime}(x) \leqslant d(x, y) \leqslant t$. Let us for a moment assume that $d_{0}^{\prime}(x)=t$. In this case, $d(x, y)=t$ must hold, and also $d(x, \tilde{y}) \geqslant t$ must be the case for all $\tilde{y} \in \Omega_{0}$. Since $\Omega_{0}$ is open, there exists a small radius $\delta>0$ such that $B_{\delta}(y) \subset \Omega_{0}$. Define $X=\mathbb{R}^{n} \backslash B_{\delta}(y)$, which is closed, and note that $x \in X$ because otherwise $x \in \Omega_{0}$ and this would lead to a contradiction with $0=d(x, x) \geqslant t>0$. Since $\partial X=\partial B_{\delta}(y) \subset \Omega_{0}$, we know that $d(x, \tilde{y}) \geqslant t$ for all $\tilde{y} \in \partial X$. This, however, implies $d(x, y)>t$ with Lemma 14 , which is a contradiction. Thus we have shown that $d_{0}^{\prime}(x)<t$ must be the case.

For the third equality, note that $\Gamma_{t}$ and $\Omega_{t}$ are clearly disjoint, so that the relation

$\Gamma_{t}=\left(\Gamma_{t} \cup \Omega_{t}\right) \backslash \Omega_{t}=\left\{x \in \mathbb{R}^{n} \mid d_{0}(x) \leqslant t\right.$ and not $\left.d_{0}^{\prime}(x)<t\right\}=\left\{x \in \mathbb{R}^{n} \mid d_{0}(x) \leqslant t \leqslant d_{0}^{\prime}(x)\right\}$

holds. Finally, assume that we know $\overline{\Omega_{0}}=\Gamma_{0} \cup \Omega_{0}$ in addition. Consider $x \in \mathbb{R}^{n}$. If $x \in \Gamma_{0} \backslash \Omega^{+}$, then $\phi(x, t)=\phi_{0}(x)=0$ and thus $x \in \Gamma_{t}$ by Theorem 2. This shows that $\Gamma_{0} \backslash \Omega^{+}$is always a subset of both sides of (11). Consider now the case $x \notin \Gamma_{0} \backslash \Omega^{+}$. For these $x$, Lemma 15 implies that $d_{0}(x)=d_{0}^{\prime}(x)$, and thus (11) holds as well.

A part of the statement of Theorem 5 can be found already in Theorem 3.2 in [19]. Note, however, that we are not aware of any actual further conclusions derived from such a HopfLax formula towards a framework for shape-sensitivity analysis or shape optimisation in general. Drawing such conclusions to derive new results in this direction is one of our main contributions, which we present in Section 5.

To conclude this section, we will now combine Theorem 5 with Theorem 2 to give formulas for the case of arbitrary signs of $F$. For this, we define $\Omega^{-}=F^{-1}((-\infty, 0))$ and $\Omega^{z}=F^{-1}(\{0\})$. We also introduce the notation

$$
\Omega_{0}^{\prime}=\mathbb{R}^{n} \backslash\left(\Gamma_{0} \cup \Omega_{0}\right)=\phi_{0}^{-1}((0, \infty))
$$

and assume for simplicity that we are in the case $\overline{\Omega_{0}}=\Gamma_{0} \cup \Omega_{0}$. Then $\mathbb{R}^{n}=\Omega_{0} \cup \Gamma_{0} \cup \Omega_{0}^{\prime}$ is a disjoint decomposition of $\mathbb{R}^{n}$ into two open sets and the interface $\Gamma_{0}$ between them, which 
has empty interior. Next, we define a modified version of $d_{0}$ from Definition 6 for this extended situation:

$$
D(x)=\left\{\begin{aligned}
\inf _{y \in \Gamma_{0} \cup \Omega_{0}} d(x, y) & \text { for } x \in \Omega^{+}, \\
-\inf _{y \in \Gamma_{0} \cup \Omega_{0}^{\prime}} d(x, y) & \text { for } x \in \Omega^{-}
\end{aligned}\right.
$$

Here, $d(\cdot, \cdot)$ is defined according to the metric discussed in Section 3 for the speed chosen as $|F| \geqslant$ 0 . Since there is no meaningful way to define $D$ on $\Omega^{z}$, we leave the distance undefined there. In the following, we never need the values of $D$ on this set. On $\Omega^{+}$, where $F$ is positive, the front moves outwards. In this case, $D$ gives the time it takes the front to reach points outside the initial geometry. For $\Omega^{-}$with negative $F$, the front moves inwards and $D$ is negative inside the initial geometry. There, $-D$ is the time until an originally interior point is hit by the front and later no longer part of $\Omega_{t}$ at all. This convention for the sign of $D$ gives it somewhat the characteristics of a signed distance function of the initial geometry (although with respect to the metric $d(\cdot, \cdot)$ induced by $|F|$ instead of the usual Euclidean distance). The composite distance $D$ defined in this way is depicted in Figure 2. Note that $D$ blows up towards $\Omega^{z}$ (the vertical line $x=0$ in the example), which is a consequence of Lemma 5. Since this corresponds to slow movement of the evolving boundary, it does not create any numerical difficulties. Take note that $D=d_{0}$ on $\Omega^{+}$by its definition in (12). On $\Omega^{-}$, the function $D$ is defined in a similar way. This implies that most of the local properties of $d_{0}$ that we will derive in the following (e. g., Lemma 16) carry over to $D$.

Corollary 1 Let $F$ be Lipschitz continuous and have compact support. Assume that $\overline{\Omega_{0}}=\Gamma_{0} \cup \Omega_{0}$ and use the notation above. Then

$$
\begin{aligned}
\Omega_{t} & =\left\{x \in \Omega^{+} \mid D(x)<t\right\} \cup\left(\Omega_{0} \cap \Omega^{z}\right) \cup\left\{x \in \Omega^{-} \mid D(x)<-t\right\}, \\
\Gamma_{t} & =\left\{x \in \Omega^{+} \mid D(x)=t\right\} \cup\left(\Gamma_{0} \cap \Omega^{z}\right) \cup\left\{x \in \Omega^{-} \mid D(x)=-t\right\}, \\
\Gamma_{t} \cup \Omega_{t} & =\left\{x \in \Omega^{+} \mid D(x) \leqslant t\right\} \cup\left(\left(\Gamma_{0} \cup \Omega_{0}\right) \cap \Omega^{z}\right) \cup\left\{x \in \Omega^{-} \mid D(x) \leqslant-t\right\}
\end{aligned}
$$

for all $t>0$. Note that we no longer require that $F \geqslant 0$ or $F \leqslant 0$ throughout $\mathbb{R}^{n}$.

Proof. Since the level-set function $\phi(x, \cdot)$ is constant in time for each $x \in \Omega^{z}$ according to Theorem 2, it is clear that the formulas hold for those $x$ and we only have to consider $x \in \Omega^{ \pm}$. Let $x \in \Omega^{+}$. Then Theorem 2 tells us that $\phi(x, t)=\phi^{+}(x, t)$, where $\phi^{+}$solves (1) with $F^{+}=\max (F, 0) \geqslant 0$. In particular, the evolving sets are the same as those generated by $\phi^{+}$ when inside of $\Omega^{+}$. Take note that $D(x)=d_{0}(x)$ in this case, since $d(x, \cdot)$ induced by $|F|$ is the same as $d(x, \cdot)$ induced by $F^{+}$using arguments based on Lemma 5 . Hence,

$$
\Omega_{t} \cap \Omega^{+}=\left\{x \in \Omega^{+} \mid d_{0}(x)<t\right\}=\left\{x \in \Omega^{+} \mid D(x)<t\right\}
$$

follows by Theorem 5 applied to $F^{+}$. The other relations follow in the same way.

It remains to consider the case $x \in \Omega^{-}$. In this situation, Theorem 2 and Lemma 4 imply that $\phi(x, t)=-\phi^{-}(x, t)$, where $\phi^{-}$solves (1) with $F^{-}=-\min (F, 0) \geqslant 0$ and initial data $-\phi_{0}$. Since this initial level-set function corresponds to the initial geometry $\Omega_{0}^{\prime}$, the distance for applying Theorem 5 is $-D(x)$ in this case. This yields

$$
x \notin \Omega_{t} \Leftrightarrow \phi(x, t) \geqslant 0 \Leftrightarrow \phi^{-}(x, t) \leqslant 0 \quad \Leftrightarrow \quad-D(x) \leqslant t \quad \Leftrightarrow \quad D(x) \geqslant-t .
$$

In other words,

$$
\Omega_{t} \cap \Omega^{-}=\left\{x \in \Omega^{-} \mid D(x)<-t\right\}
$$

when taking the complement. The same can be done for the other sets as well. 


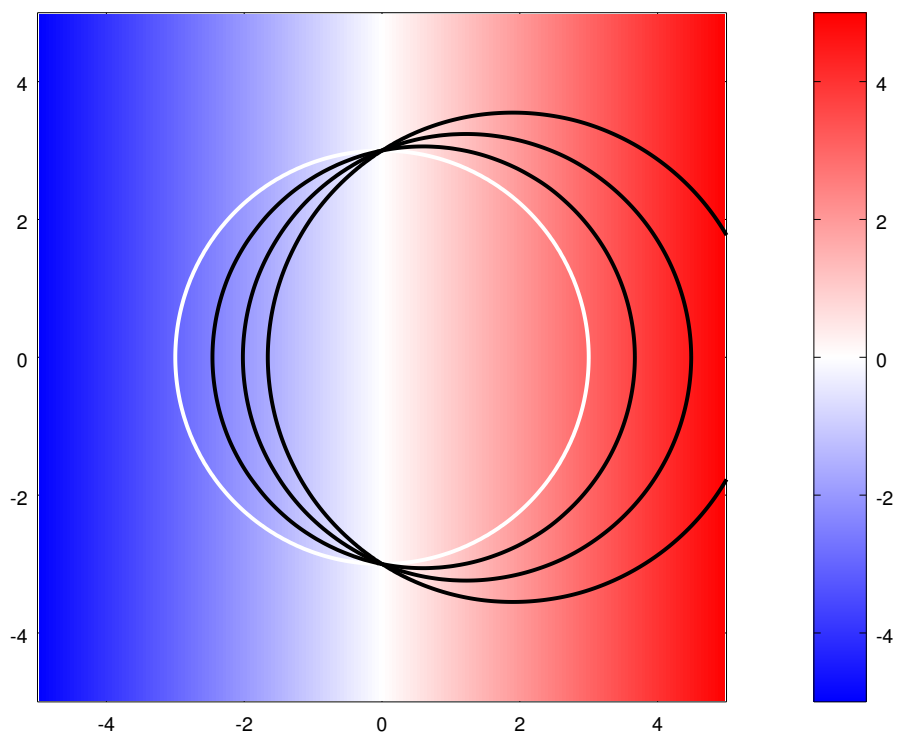

(a) The speed field, initial domain $\Omega_{0}$ (white) and resulting shape evolution (black)

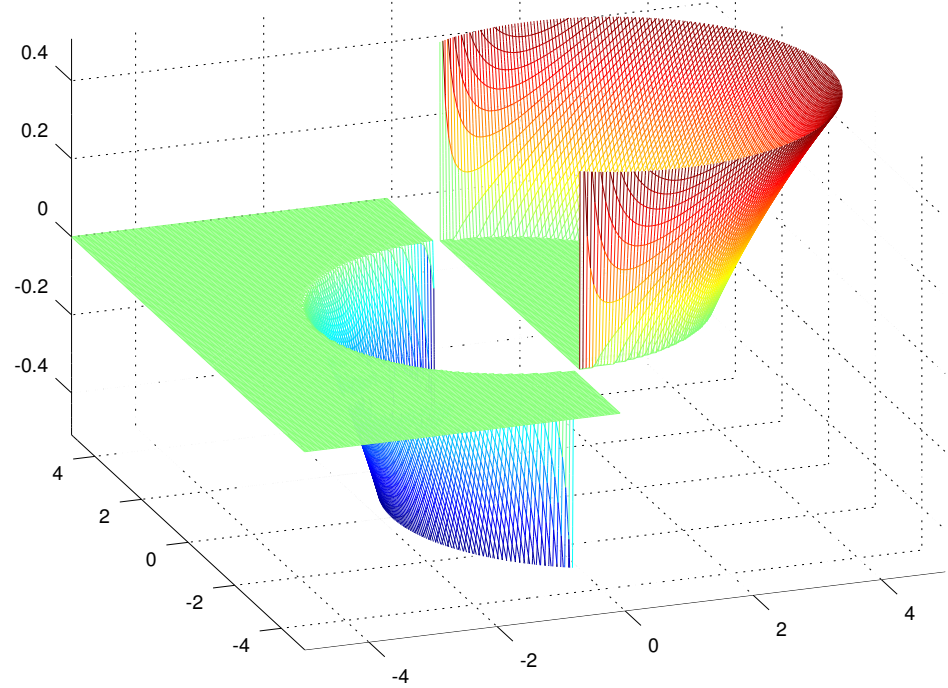

(b) The composite distance $D$ of (12)

FIG. 2: Demonstration of the composite distance $D$ of (12) and the representation formula of Corollary 1 for an example with positive and negative speeds 
The Hopf-Lax formula derived above in Corollary 1 will be used in the following section to draw some conclusions about the evolution of $\Omega_{0}$ in time. Besides these theoretical purposes, it can also be employed directly for the numerical computation of evolved domains. The distance function $D$ defined in (12) can be computed efficiently using a Fast-Marching Method (see [33] and Chapter 8 of [34]). Since one has to apply Fast Marching twice to handle arbitrary signs of the speed field, this yields a Composite Fast-Marching method. Once this is done, the evolved domains can be assembled very cheaply for arbitrary times just by using Corollary 1 . This property is useful, for instance, in the context of shape optimisation with a line search strategy: With a single computation of $D$, the evolved domain can be computed for various "trial step lengths" $t$. We employ this strategy successfully, for instance in [25]. See Section 3.5 of [27] for a more detailed description of this numerical method. Our implementation is freely available in [26].

\section{Applications}

Let us now discuss some important conclusions from and applications of our main results shown above in Theorem 5 and Corollary 1. Equipped with these powerful tools, we are now able to derive new results about non-fattening, shape-sensitivity analysis, Lipschitz continuity of the evolved levelset function $\phi$ and the effect of perturbations in the speed field or initial domain. While we believe that all of them are very interesting and important, particularly the shape calculus of Subsection 5.2 can be employed directly for level-set based shape optimisation. (See Chapter 6 of [27] for more details.)

\subsection{Measure-theoretic non-fattening}

When the level-set approach is used to describe geometries, the set $\Gamma_{t}$ as given in (7) is usually thought of as the "boundary" of the geometry one is interested in. With this interpretation, one definitely does not want $\Gamma_{t}$ to become "fat" in any way (for instance, developing interior points, or having non-zero measure). A classical result showing non-fattening in the former, topological sense under certain conditions is presented in [4]. We are not aware of any results with respect to the latter, measure-theoretic notion of non-fattening. Based on the representation of the evolving sets derived in Theorem 5, the issue of non-fattening can now be investigated with relative ease.

Lemma 16 Let $\Omega^{+}=F^{-1}((0, \infty))$ as before, $C \subset \Omega^{+}$be a connected component and assume that $d_{0}$ is finite on $C$. Then $d_{0}$ is locally Lipschitz continuous on $C$ and, in particular, differentiable almost everywhere in $C$. The same holds for $d_{0}^{\prime}$.

Proof. We can assume $F \geqslant 0$ throughout $\mathbb{R}^{n}$ without loss of generality, as we only consider $\Omega^{+}$ anyway. We also restrict ourselves to $d_{0}$ here; the same arguments can be applied for $d_{0}^{\prime}$ as well. Note that if local Lipschitz continuity is shown, differentiability almost everywhere follows by Rademacher's theorem (see Theorem 2 on page 81 of [17]).

Let $X \subset C$ be compact and convex. Since $F$ is continuous, we can introduce $F>0$ as the minimum of $F$ over $X$. We will show now that $d_{0}$ has the Lipschitz constant $L=1 / \underline{F}$ on $X$. For this, let $x, y \in X$ be given. We can choose $x_{0} \in \Gamma_{0} \cup \Omega_{0}$ with $d_{0}(x)=d\left(x, x_{0}\right)$ by Lemma 15 . Note also $d_{0}(x)<\infty$ according to our assumption, and that $d(x, y) \leqslant L|x-y|$ as shown in Lemma 11. Thus, the triangle inequality implies

$$
d_{0}(y) \leqslant d\left(y, x_{0}\right) \leqslant d(y, x)+d\left(x, x_{0}\right) \leqslant d_{0}(x)+L|x-y| .
$$


If we exchange the roles of $x$ and $y$, the same argument can be applied to derive an estimate the other way round. Taking both inequalities together, we get $\left|d_{0}(x)-d_{0}(y)\right| \leqslant L|x-y|$.

As a next step, we consider again the Eikonal equation

$$
F(x)|\nabla d(x)|=1 \text { in } C \backslash\left(\Gamma_{0} \cup \Omega_{0}\right), d(x)=0 \text { on } \Gamma_{0} \cup \Omega_{0},
$$

where $C \subset \Omega^{+}$is a connected component on which $d_{0}$ is finite. Intuitively, it makes sense that the distance $d_{0}$ of Definition 6 should solve (13) in some sense. This will be investigated in the following, because it will be a useful tool for the proof of our non-fattening result Theorem 6 . Of course, corresponding properties always also hold for $d_{0}^{\prime}$ when the boundary values are prescribed on $\overline{\Omega_{0}}$ instead of $\Gamma_{0} \cup \Omega_{0}$.

Lemma 17 The function $d_{0}$ is a viscosity supersolution of (13).

Proof. Recall that

$$
d_{0}=\inf _{y \in \Gamma_{0} \cup \Omega_{0}} d_{y}
$$

is defined as pointwise infimum of a family of functions $d_{y}(\cdot)=d(\cdot, y)$. Each $d_{y}$ is a viscosity solution of (4) according to Theorem 3 . Thus their infimum is also at least a viscosity supersolution of the equation. (See, for instance, Lemma 2.4.5 on page 101 of [20] for this well-known property of viscosity solutions.) Since $d_{0}(x) \geqslant 0$ is fulfilled for all $x \in \mathbb{R}^{n}$, it holds, in particular, for $x \in \Gamma_{0} \cup \Omega_{0}$. This shows that also the boundary condition is satisfied.

Lemma 18 The function $d_{0}$ solves (13) almost everywhere. In particular, $F(x)\left|\nabla d_{0}(x)\right|=1$ for all $x \in \Omega^{+} \backslash\left(\Gamma_{0} \cup \Omega_{0}\right)$ at which $d_{0}$ is differentiable.

Proof. Fix $x \in \Omega^{+} \backslash\left(\Gamma_{0} \cup \Omega_{0}\right)$ such that $d_{0}(x)<\infty$ and $\nabla d_{0}(x)$ exists. Note that $F(x)\left|\nabla d_{0}(x)\right| \geqslant 1$ according to Lemma 17 and thus also, in particular, $\nabla d_{0}(x) \neq 0$. We have to show $F(x)\left|\nabla d_{0}(x)\right| \leqslant 1$. Define $p_{0}=\nabla d_{0}(x) /\left|\nabla d_{0}(x)\right|$ and note that $\left|\nabla d_{0}(x)\right|=\nabla d_{0}(x) \cdot p_{0}$, which is the directional derivative of $d_{0}$ in direction $p_{0}$. For $\epsilon>0$, consider $\overline{B_{\epsilon}(x)}$. If $\epsilon$ is small enough, this is a compact and convex subset of $\Omega^{+}$, so that Lemma 16 yields that $d_{0}$ is Lipschitz continuous on $\overline{B_{\epsilon}(x)}$. The Lipschitz constant is $L_{\epsilon}=1 / \underline{F}_{\epsilon}$, where $\underline{F}_{\epsilon}=\min _{y \in \overline{B_{\epsilon}(x)}} F(y)$. By continuity of $F, L_{\epsilon} \rightarrow 1 / F(x)$ as $\epsilon \rightarrow 0^{+}$. Hence

$$
\begin{aligned}
\left|\nabla d_{0}(x)\right| & =\nabla d_{0}(x) \cdot p_{0} \\
& =\lim _{\epsilon \rightarrow 0^{+}} \frac{d_{0}\left(x+\epsilon p_{0}\right)-d_{0}(x)}{\epsilon} \leqslant \lim _{\epsilon \rightarrow 0^{+}} \frac{\left|d_{0}\left(x+\epsilon p_{0}\right)-d_{0}(x)\right|}{\epsilon} \leqslant \lim _{\epsilon \rightarrow 0^{+}} L_{\epsilon} \\
& =\frac{1}{F(x)},
\end{aligned}
$$

which completes the proof.

We now need a general lemma about the measure of level sets of Lipschitz continuous functions:

Lemma 19 Let $\Omega \subset \mathbb{R}^{n}$ and $f: \Omega \rightarrow \mathbb{R}$ be Lipschitz continuous. Then

$$
\left|f^{-1}(\{0\})\right|=\mid\{x \in \Omega \mid f(x)=0 \text { and } f \text { is differentiable at } x \text { and } \nabla f(x)=0\} \mid,
$$

where $|\cdot|$ denotes the $n$-dimensional Lebesgue measure of the preimage sets. 
Proof. This follows immediately from Lemma 7.7 on page 152 of [21].

Lemma 19 shows that if a level set of some Lipschitz continuous function "fattens" in measure, then there must also exist a set of positive measure on which its gradient exists and vanishes. This can not happen for our case of $d_{0}$, since we know that it solves (13) almost everywhere. This is the central argument in the proof of our main non-fattening result:

Theorem 6 Let $F$ be Lipschitz continuous and have compact support. Then $\left|\Gamma_{0}\right|=0$ implies $\left|\Gamma_{t}\right|=0$ for all $t \geqslant 0$.

Proof. Note first that $\left|\Gamma_{0}\right|=0$ implies, in particular, $\overline{\Omega_{0}}=\Gamma_{0} \cup \Omega_{0}$. If this were not the case, then $\Gamma_{0}$ would have interior points and thus non-zero measure. We now apply Corollary 1 to express $\Gamma_{t}$ and calculate its measure. Note that the part $\Gamma_{t} \cap \Omega^{z}=\Gamma_{0} \cap \Omega^{z}$ can be ignored, since it has zero measure by assumption. Thus, consider $\Gamma_{t} \cap \Omega^{+}=D^{-1}(\{t\})$ first. Since $D=d_{0}$ in $\Omega^{+}$, Lemma 16 and Lemma 18 apply. These results together imply that $\nabla D(x) \neq 0$ for almost all $x \in \Gamma_{t} \cap \Omega^{+}$. Consequently, it follows from Lemma 19 that $\left|\Gamma_{t} \cap \Omega^{+}\right|=0$. The same argument can also be used for $\mid \Gamma_{t} \cap \Omega^{-}$, so that we have finally shown $\left|\Gamma_{t}\right|=0$.

We conclude this subsection by using our representation formula to show non-fattening also in a topological sense. This result is similar to the classical result of [4]. Note, however, that our result concerns the sets for each instant in time separately, while the result of [4] considers the topological properties of the evolving sets in space-time. The property that we show is strictly stronger, although [4] considers a more general situation.

Theorem 7 Let $F \geqslant 0$ and assume that $\overline{\Omega_{0}}=\Gamma_{0} \cup \Omega_{0}$. Then $\overline{\Omega_{t}}=\Gamma_{t} \cup \Omega_{t}$ for all $t \geqslant 0$.

Proof. Note that $\overline{\Omega_{t}} \subset \Gamma_{t} \cup \Omega_{t}$ follows immediately from (7) because $\phi$ is continuous. Hence, we only have to show $\Gamma_{t} \cup \Omega_{t} \subset \overline{\Omega_{t}}$. Let us use Theorem 5 to express the evolving sets. The case $F(x)=0$ is easy: If $x \in \Gamma_{t} \cup \Omega_{t}$, then $x \in \Gamma_{0} \cup \Omega_{0}$ since these sets are stationary in time on $\Omega^{z}$. Hence $x \in \overline{\Omega_{0}} \subset \overline{\Omega_{t}}$ by assumption. (Recall that $F \geqslant 0$ implies monotonic growth of the domains according to Lemma 3.)

For the remaining case, let $x \in\left(\Gamma_{t} \cup \Omega_{t}\right) \cap \Omega^{+}$. Since nothing is to be shown if $x \in \Omega_{t}$, assume that $x \in \Gamma_{t}$. Thus $d_{0}(x)=t$ by Theorem 5. Lemma 15 implies that there exists $x_{0} \in \Gamma_{0} \cup \Omega_{0}$ with $d_{0}(x)=d\left(x_{0}, x\right)=t$. Assume that $x \notin \overline{\Omega_{t}}$, which means that there exists $\delta>0$ with $\overline{B_{\delta}(x)} \subset \Omega^{+} \backslash \Omega_{t}$. In other words, $d_{0}(y) \geqslant t$ for all $y \in \overline{B_{\delta}(x)}$. Note that this also implies $d\left(x_{0}, y\right) \geqslant t$ for all those $y$, since $d_{0}(y) \leqslant d\left(x_{0}, y\right)$. Consider now the closed set $X=\mathbb{R}^{n} \backslash B_{\delta}(x)$, for which we know $x_{0} \in X$ and $d\left(x_{0}, y\right) \geqslant t$ for all $y \in \partial X=\partial B_{\delta}(x)$. Thus Lemma 14 implies $d\left(x_{0}, x\right)>t$, which is a contradiction. Hence we have shown $x \in \overline{\Omega_{t}}$.

For the case of $F \leqslant 0$, a similar statement can be shown by applying Lemma 4 and taking complements of all involved sets:

$$
\left(\Gamma_{0} \cup \Omega_{0}\right)^{\circ}=\Omega_{0} \Rightarrow\left(\Gamma_{t} \cup \Omega_{t}\right)^{\circ}=\Omega_{t}
$$

It is, however, not possible to get both results at the same time, and also not to get one of them for changing sign of $F$. This is demonstrated by the following example:

EXAMPLE 1 Let $\phi_{0}(x)=|x|-1$, such that $\Omega_{0}=B_{1}(0)$ and $\Gamma_{0}=\partial \Omega_{0}$. Choose $F \leqslant 0$ with compact support and Lipschitz continuous such that $F(x)=-1$ for all $x \in B_{1+\epsilon}(0)$ with some 
$\epsilon>0$. Then $\Omega_{t}=B_{1-t}(0)$ is a shrinking circle that disappears for $t \geqslant 1$ entirely. This implies that we have, for $t=1, \Gamma_{1} \cup \Omega_{1}=\{0\} \neq \overline{\Omega_{1}}=\emptyset$.

Similarly, if we choose $\Omega_{0}$ to be $B_{2}(0) \backslash \overline{B_{1}(0)}$ and $F \geqslant 0$ with supp $(F) \subset B_{2}(0)$ and $F(x)=1$ for all $x \in B_{1+\epsilon}(0)$, then $\Omega_{t}=B_{2}(0) \backslash \overline{B_{1-t}(0)}$ and the hole disappears at $t=1$. In this case, $\left(\Gamma_{1} \cup \Omega_{1}\right)^{\circ}=B_{2}(0) \neq \Omega_{1}=B_{2}(0) \backslash\{0\}$.

\subsection{Shape sensitivity of domain functionals}

If one considers a functional depending on the evolving sets, one is often also interested in its derivative with respect to time in the shape propagation. This leads to shape derivatives, which form the foundation for level-set based schemes for shape optimisation. In the applied literature such as [14], [32] and [37], these shape-sensitivity formulas are not always rigorously justified or rely on smoothness assumptions on the domain which may not be fulfilled in practice. Based on our representation formula of Corollary 1, we are able to rigorously derive such a shape derivative for an important class of domain functionals. This result can be applied to the mentioned and other problems. In particular, our shape calculus requires no regularity assumptions on the domain $\Omega$ besides being an open set and having a boundary with measure zero. We are not aware of any other result that has this feature. Recall also that we use a scalar speed field, which defines the direction of movement via the normal direction of the domain itself. Classical shape-sensitivity analysis as discussed, for instance, in Chapter 9 of [13] usually requires a vector-valued velocity field which is completely independent of the geometry. This is a much stronger assumption than ours: Consider, for instance, the simple case $F=1$ and some initial $\Omega_{0}$ that has a corner (e. g., a square). Our approach is perfectly able to handle this situation, since the scalar speed field is obviously completely smooth in this situation. A corresponding velocity field describing the same outward movement, however, must necessarily be discontinuous at the corner due to the discontinuous normal direction there. Thus, standard shape calculus is not applicable for such a propagating geometry.

In this subsection, we will always assume that $\left|\Gamma_{t}\right|=0$ holds for all times as per Theorem 6 . For $f \in L_{\text {loc }}^{1}\left(\mathbb{R}^{n}\right)$, we define the domain functional

$$
j(t)=\int_{\Omega_{t}} f d x .
$$

With the help of the co-area formula (Theorem 2 on page 117 of [17]) and Corollary 1, the functional $j(t)$ can be expressed in terms of the composite distance $D$ defined in (12):

Theorem 8 If Corollary 1 holds for $\Omega_{t}$, then

$$
j(t)=j(0)+\int_{0}^{t} \int_{\Omega^{+} \cap D^{-1}(\{s\})} F f d \sigma d s+\int_{0}^{t} \int_{\Omega^{-} \cap D^{-1}(\{-s\})} F f d \sigma d s
$$

for all $t \geqslant 0$. Based on (12), this expression can also be written more compactly as

$$
j(t)=j(0)+\int_{-t}^{t} \int_{D^{-1}(\{s\})} F f d \sigma d s .
$$

Proof. For $t=0$, the claim is clear. So assume $t>0$ fixed now. We use the decomposition of $\Omega_{t}$ 
that is given in Corollary 1 as well as the representation

$$
j(t)=j(0)+\int_{\Omega_{t} \backslash \Omega_{0}} f d x-\int_{\Omega_{0} \backslash \Omega_{t}} f d x .
$$

Note further that

$$
\Omega_{t} \backslash \Omega_{0}=\Omega^{+} \cap D^{-1}((0, t))
$$

is the part of $\Omega_{t}$ that an outward moving boundary has created over time, while

$$
\Omega_{0} \backslash \Omega_{t}=\Omega^{-} \cap D^{-1}((-t, 0))
$$

is the subset that an inward moving boundary has removed from $\Omega_{0}$.

Consider the first of these sets now and recall that $D$ is locally Lipschitz continuous on $\Omega^{+} \cap$ $D^{-1}((0, t))$ according to Lemma 16. Furthermore, $|\nabla D(x)|=1 / F(x)$ holds for almost all $x \in$ $\Omega^{+} \cap D^{-1}((0, t))$ because of Lemma 18. Let $\left(A_{k}\right)$ be a sequence of compact subsets of $\Omega^{+} \cap$ $D^{-1}((0, t))$ converging in measure to $\Omega^{+} \cap D^{-1}((0, t))$ as $k \rightarrow \infty$. Such a sequence exists by regularity of the Lebesgue measure (Theorem 2.20 on page 50 of [31]). Since these sets are compact, $D$ is Lipschitz continuous when restricted to each $A_{k}$. We define $\chi_{k}$ to be the characteristic function of $A_{k}, \chi$ that of $\Omega^{+} \cap D^{-1}((0, t))$ and set $g_{k}=\chi_{k} F f$. Then $g_{k} \in L^{1}\left(\mathbb{R}^{n}\right)$ for each $k \in \mathbb{N}$ since $F$ has compact support. Also, $\chi_{k} \rightarrow \chi$ as $k \rightarrow \infty$ in $L^{1}\left(\mathbb{R}^{n}\right)$. Hence the co-area formula yields

$$
\int_{A_{k}} f d x=\int_{\mathbb{R}^{n}}|\nabla D| g_{k} d x=\int_{\mathbb{R}^{-1}(\{s\})} \int_{D^{-1}} F f d \sigma d s=\int_{0}^{t} \int_{A_{k} \cap D^{-1}(\{s\})} F f d \sigma d s .
$$

Using Lebesgue's dominated convergence theorem, we can pass the limit $k \rightarrow \infty$ to obtain

$$
\int_{\Omega^{+} \cap D^{-1}((0, t))} f d x=\int_{0}^{t} \int_{\Omega^{+} \cap D^{-1}(\{s\})} F f d \sigma d s .
$$

For the set $\Omega^{-} \cap D^{-1}((-t, 0))$, basically the same argument can be applied when we take the correct signs into account. As above, we proceed assuming that $D$ is Lipschitz continuous by using suitable compact cut-off sets and the dominated convergence theorem. Here, $\chi$ is the characteristic function of $\Omega^{-} \cap D^{-1}((-t, 0))$ and we define $g=\chi|F| f=-\chi F f$. Then $|\nabla D(x)|=1 /|F(x)|=-1 / F(x)$ for almost all $x \in \Omega^{-} \cap D^{-1}((-t, 0))$, since $-D$ is the solution for speed $|F|=-F$ in this part of the domain according to (12). Hence, again using the co-area formula, we get:

$$
\begin{aligned}
\int_{\Omega^{-} \cap D^{-1}((-t, 0))} f d x & =\int_{\mathbb{R}^{n}}|\nabla D| g d x=\int_{\mathbb{R}} \int_{D^{-1}(\{s\})} \chi|F| f d \sigma d s \\
& =-\int_{-t}^{0} \int_{\Omega^{-} \cap D^{-1}(\{s\})} F f d \sigma d s=-\int_{0}^{t} \int_{\Omega^{-} \cap D^{-1}(\{-s\})} F f d \sigma d s
\end{aligned}
$$

Using this now in (16) gives the correct term of (15).

As an immediate corollary of Theorem 8, the shape derivative of $j$ can be calculated in direction of a particular deformation described by a speed field $F$. This quantity is often called Eulerian derivative in the literature (see Section 2.11 of [35]). 
Corollary 2 The functional $j$ is differentiable for almost all $t \geqslant 0$ and the derivative is given by

$$
j^{\prime}(t)=\int_{\Omega^{+} \cap D^{-1}(\{t\})} F f d \sigma+\int_{\Omega^{-} \cap D^{-1}(\{-t\})} F f d \sigma .
$$

Proof. This follows by using the Lebesgue differentiation theorem (Theorem 13.15 on page 278 of [38]) on $j$ in the form of (15), where the dependence on $t$ is only in the upper bound of the onedimensional outer integral. The co-area formula guarantees that the integrand is really a function in $L^{1}(\mathbb{R})$ as is required for the differentiation theorem.

Note that the argument employed by the proof of Corollary 2 unfortunately only implies differentiability for almost all times and not full differentiability at every $t$. For this, one would have to show in addition that the derivative given in (17) can be continuously extended to all $t \geqslant 0$. We believe that this is, indeed, the case under reasonable assumptions. This question is the focus of ongoing research at the moment, and we can only refer to [28] for a partial first result. For shape optimisation based on a gradient-descent scheme, particularly $j^{\prime}(0)$ would be interesting. It is not clear by Corollary 2 alone, though, that this derivative exists. Hence, our subsequent analysis will be based on Theorem 8 instead of Corollary 2, so that we can formulate results that hold without an "almost all" qualification. These results state absolute continuity of the shape functionals. This, in turn, allows to deduce the existence of a weak almost-everywhere derivative in the same way as done in the proof of Corollary 2.

For the remainder of this subsection, we assume for simplicity that $F \geqslant 0$ is non-negative. It is straight-forward to apply the full statement of Theorem 8 in order to generalise the results to arbitrary signs of $F$. For a fixed speed field $F$, let $\Omega_{t}$ and $\Gamma_{t}$ describe the evolved domain as per Theorem 5 . We consider now a more general shape functional

$$
J(t)=J\left(\Omega_{t}\right)=\int_{\Omega_{t}} f\left(x, \Omega_{t}\right) d x .
$$

The integrand $f(\cdot, \Omega)$ is assumed to be integrable for any fixed domain $\Omega$. Furthermore, let us, for now, assume that it has a weak shape derivative $f^{\prime}$ in the sense that

$$
f\left(x, \Omega_{t}\right)=f\left(x, \Omega_{0}\right)+\int_{0}^{t} f^{\prime}\left(x, \Omega_{s}\right) d s
$$

holds for all $x \in \mathbb{R}^{n}$ and $t \geqslant 0$. The function $f^{\prime}(\cdot, \Omega)$ must also be integrable for all fixed domains $\Omega$. Under these assumptions, we can derive a total shape differential:

Corollary 3 Let $J$ and $f$ be as above. Then $J$ is absolutely continuous, i. e.,

$$
J(t)=J(0)+\int_{0}^{t} J^{\prime}(s) d s=J(0)+\int_{0}^{t}\left(\int_{\Gamma_{s}} F f\left(x, \Omega_{s}\right) d \sigma+\int_{\Omega_{s}} f^{\prime}\left(x, \Omega_{s}\right) d x\right) d s .
$$

Proof. By integrating (19) over $\Omega_{t}$, we find

$$
J\left(\Omega_{t}\right)=\int_{\Omega_{t}} f\left(x, \Omega_{0}\right) d x+\int_{\Omega_{t}} \int_{0}^{t} f^{\prime}\left(x, \Omega_{s}\right) d s d x
$$


Applying Theorem 8 to the first term (where $\Omega_{0}$ is now fixed) and Fubini's theorem to the second, this further yields

$$
J\left(\Omega_{t}\right)=J\left(\Omega_{0}\right)+\int_{0}^{t} \int_{\Gamma_{s}} F f\left(x, \Omega_{0}\right) d \sigma d s+\int_{0}^{t} \int_{\Omega_{t}} f^{\prime}\left(x, \Omega_{s}\right) d x d s .
$$

Note that this result already looks almost like the claimed (20). However, it has $\Omega_{0}$ instead of $\Omega_{s}$ in the middle term and $\Omega_{t}$ instead of $\Omega_{s}$ in the last one. Consequently, it remains to show that

$$
\int_{0}^{t} \int_{\Gamma_{s}} F\left(f\left(x, \Omega_{s}\right)-f\left(x, \Omega_{0}\right)\right) d \sigma d s=\int_{0}^{t}\left(\int_{\Omega_{t}} f^{\prime}\left(x, \Omega_{s}\right) d x-\int_{\Omega_{s}} f^{\prime}\left(x, \Omega_{s}\right) d x\right) d s .
$$

With the corresponding shape derivatives for the differences, we can turn this equation into

$$
\int_{0}^{t} \int_{\Gamma_{s}} F \int_{0}^{s} f^{\prime}\left(x, \Omega_{\tau}\right) d \tau d \sigma d s=\int_{0}^{t} \int_{s}^{t} \int_{\Gamma_{\tau}} F f^{\prime}\left(x, \Omega_{s}\right) d \sigma d \tau d s
$$

Using Fubini's theorem again on the left-hand side and renaming $s$ and $\tau$ on the right-hand side, this is further equal to

$$
\int_{0}^{t} \int_{0}^{s} \int_{\Gamma_{s}} F f^{\prime}\left(x, \Omega_{\tau}\right) d \sigma d \tau d s=\int_{0}^{t} \int_{\tau}^{t} \int_{\Gamma_{s}} F f^{\prime}\left(x, \Omega_{\tau}\right) d \sigma d s d \tau .
$$

Since both sides of this equation only express different ways to integrate over the same right triangle in the $(s, \tau)$-plane, this shows that $(21)$ and thus the claim are true.

Our result (20) matches the classical formulas for shape derivatives. Compare it, for instance, to (2.168) on page 113 of [35]. Note, however, that we were able to obtain it without employing domain transformations and without requiring regularity of the domain! To conclude this section, let us now investigate under which conditions (19) holds for a special class of shape-dependent integrands. For this, we first need a general-purpose chain rule for absolutely continuous functions:

Lemma 20 Let $f: \mathbb{R}^{k} \rightarrow \mathbb{R}$ be continuously differentiable and $g_{1}, \ldots, g_{k}: \mathbb{R} \rightarrow \mathbb{R}$ be absolutely continuous. We consider

$$
h: \mathbb{R} \rightarrow \mathbb{R}, \quad h(t)=f\left(g_{1}(t), \ldots, g_{k}(t)\right) .
$$

Then $h$ is also absolutely continuous and

$$
h^{\prime}(t)=\sum_{i=1}^{k} \partial_{i} f\left(g_{1}(t), \ldots, g_{k}(t)\right) \cdot g_{i}^{\prime}(t) .
$$

Proof. This follows from part (ii) of Theorem 4 on page 129 of [17].

In applications, it is common that the shape dependence of the integrand $f$ is due to some number of shape-dependent quantities. For instance, the integrand may depend on the volume $|\Omega|$ of the current domain or other, related values. For these integrands, we can use the results above to derive their shape derivatives as well. In particular, we are interested in integrands of the form

$$
f(x, \Omega)=f\left(x, Q_{1}(\Omega), \ldots, Q_{k}(\Omega)\right) .
$$


If the $Q$ 's have shape derivatives themselves, Lemma 20 can be used together with Corollary 3. In this situation, $J$ is again absolutely continuous with respect to $t$ and we get

$$
J(t)=J(0)+\int_{0}^{t}\left(\int_{\Gamma_{s}} F f d \sigma+\sum_{i=1}^{k} \int_{\Omega_{s}} \partial_{i} f \cdot Q_{i}^{\prime} d x\right) d s .
$$

Thus, if all the $Q$ 's are domain functionals of the form (18), (23) themselves, we can recursively apply (24) to find shape derivatives. As long as there are no circular dependencies among the various shape-dependent quantities (i. e., the dependency graph is a tree), this process will work fine.

\subsection{Lipschitz continuity with optimal constants}

It is a well-known fact that viscosity solutions of an initial-value problem (like the level-set equation (1)) often preserve Lipschitz continuity of the initial function $\phi_{0}$. Usually, this property is deduced from the comparison principle. See, for instance, Theorem 3.5.1 on page 139 of [20] or the related result in [24] for bounded domains. Following a slightly different route, we can also use our representation formula (9) to show Lipschitz continuity of $\phi$ both in time (see Theorem 9) and spatially (in Theorem 11). Based on the construction given in Example 2, we can even demonstrate that our results are sharp.

Before we can show Lipschitz continuity of $\phi$ in time, we have to consider how admissible points in the minimum of (9) change if the upper bound $t$ is modified.

Lemma 21 Let $F(x) \geqslant 0$ for some $x \in \mathbb{R}^{n}$. Assume that $|F| \leqslant \bar{F}, x^{t} \in \mathbb{R}^{n}$ and $s, t \geqslant 0$ with $d\left(x, x^{t}\right) \leqslant t$. Then there exists $x^{s} \in \mathbb{R}^{n}$ with $d\left(x, x^{s}\right) \leqslant s$ and $\left|x^{s}-x^{t}\right| \leqslant \bar{F}|t-s|$.

Proof. Consider first the trivial case $s=0$ : We can pick $x^{s}=x$ and know by Lemma 8 that

$$
\left|x^{s}-x^{t}\right|=\left|x-x^{t}\right| \leqslant \bar{F} \cdot d\left(x, x^{t}\right) \leqslant \bar{F} t=\bar{F}|t-0| .
$$

Thus, assume now $s>0$. Similarly, also the case $s \geqslant d\left(x, x^{t}\right)$ is trivial, as one can choose $x^{s}=x^{t}$. Consequently, we assume further $s<d\left(x, x^{t}\right) \leqslant t$ from now on. Choose a minimising path $\xi \in$ $X_{\text {ad }}\left(x, x^{t}\right)$ with $l(\xi)=d\left(x, x^{t}\right)$. Since $\xi$ and $d$ are continuous, we know that $\tau \mapsto d(x, \xi(\tau))$ is continuous as well and ranges from zero at $\tau=0$ to $d\left(x, x^{t}\right)>s$ at $\tau=1$. Thus, the intermediatevalue theorem implies the existence of $\tau_{0} \in(0,1)$ and $x^{s}=\xi\left(\tau_{0}\right)$ with $d\left(x, x^{s}\right)=s$. Denote by $\tilde{\xi}$ the part of $\xi$ between $x^{s}$ and $x^{t}$, i. e., for times in $\left[\tau_{0}, 1\right]$. Then we get

$$
d\left(x, x^{s}\right)+l(\tilde{\xi})=s+l(\tilde{\xi}) \leqslant l(\xi) \leqslant t
$$

since $d\left(x, x^{s}\right)$ is the shortest distance between $x$ and $x^{s}$, while the initial part of $\xi$ is just a particular path. Hence we get also

$$
l(\tilde{\xi}) \leqslant t-s=|t-s|,
$$

which further implies that

$$
\left|x^{s}-x^{t}\right| \leqslant \bar{F} \cdot l(\tilde{\xi}) \leqslant \bar{F}|t-s|
$$


Theorem 9 Let $|F| \leqslant \bar{F}$ on $\mathbb{R}^{n}$. Then

$$
|\phi(x, s)-\phi(x, t)| \leqslant L_{\phi_{0}} \bar{F} \cdot|t-s|
$$

for all $x \in \mathbb{R}^{n}$ and $s, t \geqslant 0$.

Proof. Let $x \in \mathbb{R}^{n}$ and $s, t \geqslant 0$. If $F(x)=0$, then $\phi(x, t)=\phi(x, s)=\phi_{0}(x)$, thus this case is trivial. If $F(x)<0$, we can use Lemma 4 to reduce the situation to the case of $F(x)>0$. Thus, assume $F(x)>0$ without loss of generality now. Pick $x^{t}$ as minimiser of (9), such that $\phi(x, t)=\phi_{0}\left(x^{t}\right)$ and $d\left(x, x^{t}\right) \leqslant t$. Then, using Lemma 21 and the Lipschitz continuity of $\phi_{0}$, we get

$$
\phi(x, s) \leqslant \phi_{0}\left(x^{s}\right) \leqslant \phi_{0}\left(x^{t}\right)+L_{\phi_{0}}\left|x^{s}-x^{t}\right| \leqslant \phi(x, t)+L_{\phi_{0}} \bar{F} \cdot|s-t| .
$$

Using a symmetric argument with $s$ and $t$ exchanged completes the proof.

Next, we can show spatial Lipschitz continuity in terms of the distance $d$. This is a consequence of (9) and Lipschitz continuity in time. Note that $d$ is itself Lipschitz continuous where $F$ is bounded away from zero (recall Lemma 11). Consequently, Theorem 10 actually gives a Lipschitz constant that is uniform for all times in these cases. However, where $F$ may become zero or change its sign, this result makes no statement and the subsequent Theorem 11 must be applied instead.

Theorem 10 Let $x, y \in \mathbb{R}^{n}, t \geqslant 0$ and $|F| \leqslant \bar{F}$. Denote by $L=L_{\phi_{0}} \bar{F}$ the temporal Lipschitz constant of $\phi$ according to Theorem 9. Then

$$
|\phi(x, t)-\phi(y, t)| \leqslant L \cdot d(x, y) .
$$

Proof. The claim is trivial if $x=y$, so assume $x \neq y$. Moreover, if $F(x)=0, F(y)=0$ or they have differing signs, then $d(x, y)=\infty$ and nothing is to be shown. The case $F(x), F(y)<0$ can be reduced to $F(x), F(y)>0$ with the help of Lemma 4, so assume $F(x), F(y)>0$ from now on. For the trivial case of $t=0$, we get

$$
|\phi(x, 0)-\phi(y, 0)|=\left|\phi_{0}(x)-\phi_{0}(y)\right| \leqslant L_{\phi_{0}}|x-y| \leqslant L_{\phi_{0}} \bar{F} \cdot d(x, y),
$$

where the last estimate is due to Lemma 8.

Consider now $t>0$ and note that $\phi$ is given by (9). If we choose $x^{t}$ and $y^{t}$ as minimisers for $\phi(x, t)$ and $\phi(y, t)$, respectively, we get

$$
\phi(x, t)=\phi_{0}\left(x^{t}\right), \quad \phi(y, t)=\phi_{0}\left(y^{t}\right), \quad \max \left(d\left(x, x^{t}\right), d\left(y, y^{t}\right)\right) \leqslant t .
$$

Define $s=d\left(x, y^{t}\right)$ and note that our assumption of Lipschitz continuity of $\phi$ in time gives

$$
\phi(x, t)-L|t-s| \leqslant \phi(x, s) \leqslant \phi_{0}\left(y^{t}\right)=\phi(y, t),
$$

so that further

$$
\phi(x, t) \leqslant \phi(y, t)+L\left|t-d\left(x, y^{t}\right)\right| .
$$

Consider first the case $d\left(x, y^{t}\right) \geqslant t$. Then

$$
\left|t-d\left(x, y^{t}\right)\right|=d\left(x, y^{t}\right)-t \leqslant d(x, y)+d\left(y, y^{t}\right)-t \leqslant d(x, y),
$$


which gives

$$
\phi(x, t) \leqslant \phi(y, t)+L \cdot d(x, y) .
$$

In the second case of $d\left(x, y^{t}\right)<t$, we get

$$
\phi(x, t) \leqslant \phi_{0}\left(y^{t}\right)=\phi(y, t) \leqslant \phi(y, t)+L \cdot d(x, y)
$$

since $y^{t}$ is admissible also for $x$ in (9). If we repeat this argument now with $x$ and $y$ exchanged, the claimed Lipschitz continuity follows.

Let us continue with the final goal of deriving a spatial Lipschitz constant with respect to the usual Euclidean distance $|x-y|$. As a first step towards this result, we can show it in the case that $F(x)=0$ holds at least for one of the two points involved. This is a very important piece of information, as it complements the earlier result in Theorem 10, which handles the situation within the support of $F$.

Lemma 22 Let $x, y \in \mathbb{R}^{n}, t \geqslant 0$ and assume that $F(x)=0$. If $y^{t} \in \mathbb{R}^{n}$ realises the minimum in (9) for $\phi(y, t)$, then

$$
\left|y^{t}-y\right| \leqslant\left(e^{L_{F} t}-1\right)|x-y|
$$

and, furthermore,

$$
|\phi(x, t)-\phi(y, t)| \leqslant L_{\phi_{0}} e^{L_{F} t} \cdot|x-y| .
$$

Proof. If $F(y)=0$, then $y^{t}=y$ is the minimiser of (9), which makes the first estimate trivial. The same is true if $t=0$. For $F(y)<0$, we can use Lemma 4 to convert the situation to the remaining case of $F(y)>0$ as before. Note that $F(y) \leqslant F(x)+L_{F}|x-y|=L_{F}|x-y|$. Combining this with the first estimate of Lemma 9 yields

$$
\left|y-y^{t}\right| \leqslant \frac{F(y)}{L_{F}}\left(e^{L_{F} t}-1\right) \leqslant\left(e^{L_{F} t}-1\right)|x-y| .
$$

For the second part, we use this result in combination with (9) to get

$$
\begin{aligned}
|\phi(x, t)-\phi(y, t)| & =\left|\phi_{0}(x)-\phi_{0}\left(y^{t}\right)\right| \leqslant L_{\phi_{0}}\left(|x-y|+\left|y-y^{t}\right|\right) \\
& \leqslant L_{\phi_{0}}\left(|x-y|+\left(e^{L_{F} t}-1\right)|x-y|\right)=L_{\phi_{0}} e^{L_{F} t} \cdot|x-y| .
\end{aligned}
$$

Lemma 23 Let $x, y \in \Omega^{+}$and $t>0$. Assume that $\phi(y, t)=\phi_{0}\left(y^{t}\right)$ with $d\left(y, y^{t}\right) \leqslant t$. Then there exists $x^{\prime} \in \mathbb{R}^{n}$ with $d\left(x, x^{\prime}\right) \leqslant t$ and $\left|x^{\prime}-y^{t}\right| \leqslant e^{L_{F} t}|x-y|$.

Proof. If $d\left(x, y^{t}\right) \leqslant t$, we can choose $x^{\prime}=y^{t}$. Also, if $F(y) \leqslant L_{F}|x-y|$, we can use $x^{\prime}=x$. In this situation, the first estimate in Lemma 9 gives

$$
\begin{aligned}
\left|x^{\prime}-y^{t}\right| & \leqslant|x-y|+\left|y-y^{t}\right| \\
& \leqslant|x-y|+\frac{F(y)}{L_{F}}\left(e^{L_{F} t}-1\right) \\
& \leqslant|x-y| \cdot\left(1+e^{L_{F} t}-1\right)=e^{L_{F} t}|x-y| .
\end{aligned}
$$




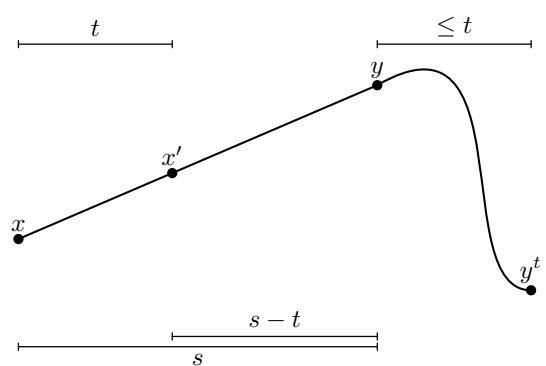

(a) The case $t<s$

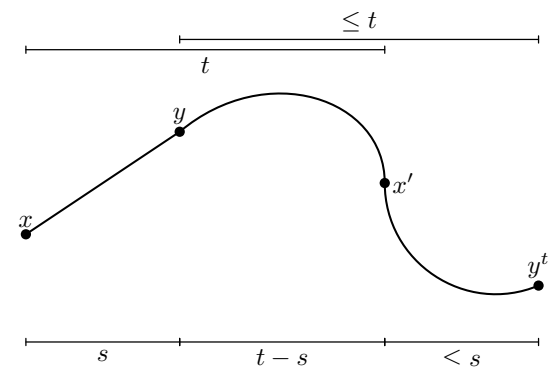

(b) The case $t>s$

FIG. 3: Sketches for the situations in the proof of Lemma 23. Indicated is always the path length according to $l$ in Definition 3.

Thus consider now the case $d\left(x, y^{t}\right)>t$ and $F(y)>L_{F}|x-y|$. Let $s=l\left(S_{x y}\right)$ denote the path length of the straight line $S_{x y}$ from $x$ to $y$. The third estimate in Lemma 9 implies

$$
|x-y| \geqslant \frac{F(y)}{L_{F}}\left(1-e^{-s L_{F}}\right),
$$

which is equivalent to

$$
\left(1-e^{-s L_{F}}\right) \leqslant \frac{L_{F}|x-y|}{F(y)} .
$$

Apply Lemma 7 to choose $\xi_{y} \in X_{\text {ad }}\left(y, y^{t}\right)$ with $l\left(\xi_{y}\right)=d\left(y, y^{t}\right) \leqslant t$. We will construct $x^{\prime}$ on the path $\xi$ that is formed by first following $S_{x y}$ from $x$ to $y$ and then moving along $\xi_{y}$ from $y$ to $y^{t}$. Note that $S_{x y}$ is entirely inside of $\Omega^{+}$since $F(y)>L_{F}|x-y|$ and

$$
F\left(S_{x y}(\tau)\right)=F(x+\tau(y-x)) \geqslant F(y)-L_{F}(1-\tau)|x-y|>0
$$

for arbitrary $\tau \in[0,1]$. The path $\xi$ can be expressed explicitly as

$$
\xi(\tau)=\left\{\begin{aligned}
S_{x y}(2 \tau) & \text { for } \tau \in[0,1 / 2], \\
\xi_{y}(2 \tau-1) & \text { for } \tau \in[1 / 2,1] .
\end{aligned}\right.
$$

Denote for a moment the length of $\xi$ restricted to $[0, \tau]$ by $\lambda(\tau)$ and note that $\lambda$ is continuous. Since $\lambda(1)=l(\xi) \geqslant d\left(x, y^{t}\right)>t$ and $\lambda(0)=0<t$, we can find $\tau_{0} \in(0,1)$ with $\lambda\left(\tau_{0}\right)=t$. Choose $x^{\prime}=\xi\left(\tau_{0}\right)$, so that $d\left(x, x^{\prime}\right) \leqslant \lambda\left(\tau_{0}\right)=t$. It remains to show $\left|x^{\prime}-y^{t}\right| \leqslant e^{L_{F} t}|x-y|$. For this, we have to consider two cases depending on which segment of $\xi$ the point $x^{\prime}$ comes to lie on. The path $\xi$ is sketched for both situations in Figure 3.

If $\tau_{0} \leqslant 1 / 2$, then $x^{\prime}$ is still part of the straight initial piece of $\xi$ as shown in Figure 3a. This means that $t \leqslant s=l\left(S_{x y}\right)$ as well as $|x-y|=\left|x-x^{\prime}\right|+\left|x^{\prime}-y\right|$. Equality holds here because $x, x^{\prime}$ and $y$ are collinear. Since the path length from $x^{\prime}$ to $y$ on $S_{x y}$ is the remaining $s-t$ and thus also, in particular, $d\left(x^{\prime}, y\right) \leqslant s-t$, we can again employ Lemma 9 to find

$$
\left|x^{\prime}-y\right| \leqslant \frac{F\left(x^{\prime}\right)}{L_{F}}\left(e^{L_{F} d\left(x^{\prime}, y\right)}-1\right) \leqslant \frac{F\left(x^{\prime}\right)}{L_{F}}\left(e^{(s-t) L_{F}}-1\right) .
$$


Together with Lipschitz continuity, this yields

$$
F(y) \leqslant F\left(x^{\prime}\right)+L_{F}\left|x^{\prime}-y\right| \leqslant F\left(x^{\prime}\right)\left(1+e^{(s-t) L_{F}}-1\right)=F\left(x^{\prime}\right) e^{(s-t) L_{F}}
$$

and, consequently, $F\left(x^{\prime}\right) \geqslant F(y) e^{(t-s) L_{F}}$. Furthermore, since

$$
F\left(x^{\prime}\right) \geqslant F(y)-L_{F}\left|x^{\prime}-y\right|>L_{F}\left(|x-y|-\left|x^{\prime}-y\right|\right)=L_{F}\left|x^{\prime}-x\right|,
$$

the third estimate of Lemma 9 is applicable again and gives

$$
\left|x^{\prime}-x\right| \geqslant \frac{F\left(x^{\prime}\right)}{L_{F}}\left(1-e^{-t L_{F}}\right) \geqslant \frac{F(y)}{L_{F}}\left(e^{(t-s) L_{F}}-e^{-s L_{F}}\right)=\frac{F(y)}{L_{F}}\left(e^{L_{F} t}-1\right) e^{-s L_{F}} .
$$

All together, we have

$$
\begin{aligned}
\left|x^{\prime}-y^{t}\right| & \leqslant\left|x^{\prime}-y\right|+\left|y-y^{t}\right|=|x-y|+\left|y-y^{t}\right|-\left|x-x^{\prime}\right| \\
& \leqslant|x-y|+\frac{F(y)}{L_{F}}\left(e^{L_{F} t}-1\right)-\frac{F(y)}{L_{F}}\left(e^{L_{F} t}-1\right) e^{-s L_{F}} \\
& =|x-y|+\frac{F(y)}{L_{F}}\left(e^{L_{F} t}-1\right)\left(1-e^{-s L_{F}}\right) \\
& \leqslant|x-y|+\frac{F(y)}{L_{F}}\left(e^{L_{F} t}-1\right) \frac{L_{F}|x-y|}{F(y)}=e^{L_{F} t}|x-y|,
\end{aligned}
$$

which finishes the proof for this case. The last estimate is due to (25).

Now consider $\tau_{0} \geqslant 1 / 2$, which means that $t \geqslant s$ and that $x^{\prime}$ lies on $\xi_{y}$ between $y$ and $y^{t}$. Take a look at Figure 3b. Consequently, if we consider the piece of $\xi_{y}$ between $y$ and $x^{\prime}$ (for times in $\left.\left[1 / 2, \tau_{0}\right]\right)$, its path length is $t-s \geqslant 0$. Since $y^{t}=\xi_{y}(1)$ and $l\left(\xi_{y}\right) \leqslant t$, we know that the length of the remaining piece of $\xi_{y}$ between $x^{\prime}$ and $y^{t}$ is at most $s$. Thus

$$
\left|x^{\prime}-y^{t}\right| \leqslant \frac{F\left(x^{\prime}\right)}{L_{F}}\left(e^{L_{F} s}-1\right)=\frac{F\left(x^{\prime}\right)}{L_{F}} e^{s L_{F}}\left(1-e^{-s L_{F}}\right)
$$

by Lemma 9. Using (25), this yields

$$
\left|x^{\prime}-y^{t}\right| \leqslant \frac{F\left(x^{\prime}\right)}{L_{F}} e^{s L_{F}} \frac{L_{F}|x-y|}{F(y)}=\frac{F\left(x^{\prime}\right)}{F(y)} e^{s L_{F}}|x-y| .
$$

Similarly to the last case and (26), we can combine Lemma 9 and the Lipschitz continuity of $F$ to obtain

$$
F\left(x^{\prime}\right) \leqslant F(y)+L_{F}\left|x^{\prime}-y\right| \leqslant F(y) e^{(t-s) L_{F}},
$$

which allows us to rewrite (27) to

$$
\left|x^{\prime}-y^{t}\right| \leqslant e^{t L_{F}}|x-y|
$$

Now we have everything together to show spatial Lipschitz continuity: 
Theorem 11 For all $x, y \in \mathbb{R}^{n}$ and $t \geqslant 0$, we have the Lipschitz estimate

$$
|\phi(x, t)-\phi(y, t)| \leqslant L_{\phi_{0}} e^{L_{F} t} \cdot|x-y| .
$$

Proof. If $F(x)=0$ or $F(y)=0$, the result follows from Lemma 22. If $F(x)$ and $F(y)$ have different signs, we can split the straight line $S_{x y}$ between $x$ and $y$ at some point $z$ that has $F(z)=0$, use Theorem 2 and apply Lemma 22 twice to get the claimed Lipschitz continuity. Also, if $t=0$, the result follows since $\phi(\cdot, 0)=\phi_{0}$ is Lipschitz continuous. Thus it remains to consider, without loss of generality, the case $F(x), F(y)>0$ and $t>0$. Let $y^{t} \in \mathbb{R}^{n}$ with $d\left(y, y^{t}\right) \leqslant t$ and $\phi(y, t)=\phi_{0}\left(y^{t}\right)$ be a minimiser of (9). Using Lemma 23, we get $x^{\prime} \in \mathbb{R}^{n}$ with $d\left(x, x^{\prime}\right) \leqslant t$ and $\left|x^{\prime}-y^{t}\right| \leqslant e^{L_{F} t}|x-y|$. It follows that

$$
\phi(x, t) \leqslant \phi_{0}\left(x^{\prime}\right) \leqslant \phi_{0}\left(y^{t}\right)+L_{\phi_{0}}\left|x^{\prime}-y^{t}\right| \leqslant \phi(y, t)+L_{\phi_{0}} e^{L_{F} t} \cdot|x-y|,
$$

which gives the claimed result when the same argument is applied again with $x$ and $y$ exchanged.

We will now conclude this subsection with an example that demonstrates that the constants given in Theorem 9 and Theorem 11 are sharp:

EXAMPLE 2 Let $L_{\phi_{0}}, L_{F}, a>0$ be given. We define $\phi_{0}: \mathbb{R} \rightarrow \mathbb{R}$ by

$$
\phi_{0}(x)=\left\{\begin{aligned}
0 & \text { if } x \leqslant 0, \\
-L_{\phi_{0}} x & \text { for } x \in[0,2 a], \\
-2 a L_{\phi_{0}} & \text { if } x \geqslant 2 a
\end{aligned}\right.
$$

as well as $F: \mathbb{R} \rightarrow \mathbb{R}$ by

$$
F(x)=\left\{\begin{aligned}
L_{F} x & \text { for } x \in[0, a] \\
L_{F}(2 a-x) & \text { for } x \in[a, 2 a] \\
0 & \text { else. }
\end{aligned}\right.
$$

These functions are sketched in Figure 4. Note that $\phi_{0}$ and $F$ are Lipschitz continuous with Lipschitz constants $L_{\phi_{0}}$ and $L_{F}$, respectively, $F$ has compact support on $[0,2 a]$ and that $F \geqslant 0$. Furthermore,

$$
|F(x)| \leqslant \bar{F}=a L_{F}
$$

for all $x \in \mathbb{R}$. This means that the parameter $a$ can be used to choose the maximal value $\bar{F}$ of $F$ independently of the Lipschitz constants. Thus all quantities that appear in the proven Lipschitz constants can be influenced by the parameters in this example. This situation fulfils all assumptions we have made for the theoretical considerations above, so that our results apply here. If we denote the solution of (1) by $\phi$ as usual, Theorem 4 holds and thus $\phi$ is given by (9).

If $x$ or $y$ are not in $(0,2 a)$, then clearly $d(x, y)=\infty$ if $x \neq y$ and $d(x, y)=0$ for $x=y$. In the case $x, y \in(0,2 a)$, we have

$$
d(x, y)=\left|\int_{x}^{y} \frac{1}{F(\xi)} d \xi\right| .
$$

Note that there is no real choice for different paths in one dimension. The absolute value ensures that the expression is correct even for $y<x$, when the integral itself is negative. Also note that 


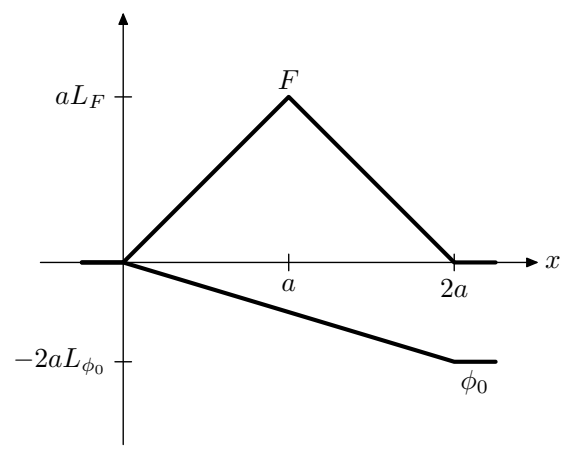

FIg. 4: The situation of Example 2

$d(x, y) \rightarrow \infty$ for $y \rightarrow 2 a^{-}$and that $\phi_{0}$ is strictly decreasing on [0,2a]. This implies that for $x \in(0,2 a)$ and $t \geqslant 0$, the minimiser of (9) is always the unique $x^{t} \in[x, 2 a)$ with $d\left(x, x^{t}\right)=t$. Assume $x \in(0, a]$ and $x^{\prime} \in[x, a]$. Then $F(\xi)=L_{F} \xi$ for $\xi \in\left[x, x^{\prime}\right]$ and we can solve the integral to get

$$
d\left(x, x^{\prime}\right)=\int_{x}^{x^{\prime}} \frac{1}{L_{F} \xi} d \xi=\frac{\log x^{\prime}-\log x}{L_{F}} .
$$

Thus, if $t \leqslant d(x, a)$, we know that $x^{t} \in[x, a]$ can be found by solving $d\left(x, x^{t}\right)=t$ together with (28) for the unknown $x^{t}$. It is trivial to see that the result is $x^{t}=x e^{L_{F} t}$. Therefore, we have shown that for every $x \in(0, a)$ and $t \geqslant 0$ small enough, the evolved level-set function is given by

$$
\phi(x, t)=\phi_{0}\left(x^{t}\right)=\phi_{0}\left(x e^{L_{F} t}\right)=-L_{\phi_{0}} x e^{L_{F} t} .
$$

We will now take derivatives of (29) in order to verify that this solution does, indeed, realise the Lipschitz constants we have shown. Note that for arbitrarily large $t$, there always exists $x \in(0, a)$ with $t<d(x, a)$ so that (29) can be applied. Taking the derivative with respect to $x$ shows that the maximum Lipschitz constant according to Theorem 11 is, indeed, tested with this example. For the time derivative, we get

$$
\left|\frac{\partial \phi}{\partial t}(x, t)\right|=L_{\phi_{0}} L_{F} x e^{L_{F} t}
$$

as lower bound on the Lipschitz constant, which is valid at least for every $x \in(0, a)$ and $0 \leqslant t \leqslant$ $d(x, a)$. Clearly the largest bound is achieved if $x$ is as large as possible, which means just so large that $t=d(x, a)$. By taking (28) into account, this is at $x=a e^{-L_{F} t}$. Consequently, the temporal Lipschitz constant must be at least

$$
L_{\phi_{0}} L_{F} a e^{-L_{F} t} e^{L_{F} t}=a L_{F} L_{\phi_{0}}=\bar{F} L_{\phi_{0}} .
$$

This matches the result from Theorem 9.

\subsection{Propagation speed of perturbations}

Since $F$ in the level-set equation (1) describes a speed of movement, it is intuitive to assume that the maximal speed $\bar{F}$ is also the maximal speed with which perturbations in the initial geometry and/or 
the speed field itself can propagate. With the help of the representation formula (9), this result can be proven easily. We will assume here that a perturbation happens on some set $A \subset \mathbb{R}^{n}$ and that we consider a point $x \notin A$ with Euclidean distance $\delta>0$ to $A$, i. e.,

$$
\delta=\inf _{y \in A}|x-y|>0 .
$$

The first result concerns perturbations in the initial geometry:

Lemma 24 Assume $0 \leqslant F \leqslant \bar{F}$. Let $\phi_{0}$ and $\tilde{\phi}_{0}$ be two initial level-set functions and $\phi, \tilde{\phi}$ the corresponding solutions of (1) for the same $F$ in both cases. Assume that $\phi_{0}(x)=\tilde{\phi}_{0}(x)$ for all $x \notin A$, and that $x \in \mathbb{R}^{n} \backslash \bar{A}$ is given with $\delta>0$ defined according to (30). Then $\phi(x, t)=\tilde{\phi}(x, t)$ for all $t<\delta / \bar{F}$.

Proof. We may assume that $F(x)>0$ because $\phi(x, t)=\phi_{0}(x)=\tilde{\phi}_{0}(x)=\tilde{\phi}(x, t)$ otherwise, which makes the statement trivial. From Lemma 8 we know that $|x-y| \leqslant \bar{F} \cdot d(x, y)$ for all $y \in \mathbb{R}^{n}$. In particular, this implies for all $y \in A$ :

$$
\delta \leqslant|x-y| \leqslant \bar{F} \cdot d(x, y) \Rightarrow d(x, y) \geqslant \frac{\delta}{\bar{F}}
$$

Choose now $t<\delta / \bar{F}$ and $z$ with $d(x, z) \leqslant t$. It follows that $z \notin A$ and thus $\phi_{0}(z)=\tilde{\phi}_{0}(z)$. The claim follows now using the form (9) for the solutions as implied by Theorem 4 .

Next, we consider what happens when the same initial geometry propagates with two different speed fields $F$ and $\tilde{F}$ :

Lemma 25 Let $0 \leqslant F, \tilde{F} \leqslant \bar{F}$ be two different speed fields. Assume furthermore that $F(x)=\tilde{F}(x)$ for all $x \notin A$ and let $\phi_{0}$ describe some initial geometry. We denote by $d$ and $\tilde{d}$ the distances induced by $F$ and $\tilde{F}$, respectively, and by $\phi$ and $\tilde{\phi}$ the solutions of (1) for both speed fields with the same initial function $\phi_{0}$. For $x \in \mathbb{R}^{n} \backslash \bar{A}$, let $\delta>0$ be as in (30). Then $\phi(x, t)=\tilde{\phi}(x, t)$ for all $t<\delta / \bar{F}$.

Proof. The claim is clear if $F(x)=0$, so assume $F(x)>0$. Let $t<\delta / \bar{F}$ be given. Since $x \notin A$, this also implies $\tilde{F}(x)=F(x)>0$. We want to show that

$$
\left\{y \in \mathbb{R}^{n} \mid d(x, y) \leqslant t\right\}=\left\{y \in \mathbb{R}^{n} \mid \tilde{d}(x, y) \leqslant t\right\},
$$

which then implies the claim via (9) and Theorem 4. Choose $y \in \mathbb{R}^{n}$ with $d(x, y) \leqslant t<\delta / \bar{F}$. Let $\xi \in X_{\text {ad }}(x, y)$ be some admissible path with $l(\xi)<\delta / \bar{F}$. Assume there exists $t_{0} \in[0,1]$ with $z=\xi\left(t_{0}\right) \in A$. But then $l(\xi) \geqslant d(x, z)+d(z, y)$ and

$$
d(x, z) \geqslant \frac{|x-z|}{\bar{F}} \geqslant \frac{\delta}{\bar{F}}
$$

by Lemma 8, which is a contradiction. Thus $\xi$ never touches $A$ and, consequently, $l(\xi)=\tilde{l}(\xi)$. This implies that every (short enough) path contributing to the infimum for $d(x, y)$ is also admissible for $\tilde{d}(x, y)$ with the same length. Hence $\tilde{d}(x, y) \leqslant d(x, y)$, showing inclusion from left to right in (31). The inclusion from right to left works just the same.

As a final result, let us combine Lemma 24 and Lemma 25 into a single theorem: 
Theorem 12 Let $|F|,|\tilde{F}| \leqslant \bar{F}$ and $\phi_{0}$, $\tilde{\phi}_{0}$ be two initial level-set functions. Denote the corresponding solutions of (1) by $\phi$ and $\tilde{\phi}$, respectively. Assume that $F(x)=\tilde{F}(x)$ and $\phi_{0}(x)=$ $\tilde{\phi}_{0}(x)$ for all $x \notin A$. Then for each $x \in \mathbb{R}^{n} \backslash A$ with $\delta>0$ defined as per (30), we have $\phi(x, t)=\tilde{\phi}(x, t)$ for all $t \leqslant \delta / \bar{F}$.

Proof. It is enough to consider $x \in \mathbb{R}^{n} \backslash \bar{A}$ and $t<\delta / \bar{F}$ since $\phi$ and $\tilde{\phi}$ are continuous. Thus, let $x \in \mathbb{R}^{n} \backslash \bar{A}$ and $t<\delta / \bar{F}$. Note that $F(x)=\tilde{F}(x)$ and that we can reduce the general case to that of $F, \tilde{F} \geqslant 0$ by using Theorem 2. We introduce an "intermediate solution" $\hat{\phi}$ as the solution of (1) with $F$ and $\tilde{\phi}_{0}$. Lemma 24 implies that $\hat{\phi}(x, t)=\phi(x, t)$. Furthermore, Lemma 25 implies also $\hat{\phi}(x, t)=\tilde{\phi}(x, t)$, so that the claim is shown.

Note also that the upper bound $\delta / \bar{F}$ can be further improved if necessary: Instead of estimating $F$ and $\tilde{F}$ very roughly by $\bar{F}$, we can define

$$
d(x, A)=\inf _{y \in A} d(x, y)=\inf _{y \in A} \tilde{d}(x, y) .
$$

Equality between the definition with $d$ and that with $\tilde{d}$ is due to Lemma 14, which implies that the shortest paths must be outside of $A$. Following the proof of Theorem 12 closely, one can see that it remains true for all $t \leqslant d(x, A)$. Since $d(x, A)>\delta / \bar{F}$ in general, this leads to a stronger statement.

Acknowledgements. The author would like to thank Wolfgang Ring of the University of Graz for fruitful discussions about this topic and thorough proof-reading of the manuscript. This work is supported by the Austrian Science Fund (FWF) and the International Research Training Group IGDK 1754.

\section{References}

1. BARdi, M., \& CAPUZzo-DolcetTA, I., Optimal Control and Viscosity Solutions of Hamilton-JacobiBellman Equations. Systems \& Control: Foundations \& Applications. Birkhäuser, 1997. Zb10890 49011 MR1484411

2. BARDi, M. \& Evans, L. C., On Hopf's Formulas for Solutions of Hamilton-Jacobi Equations. Nonlinear Analysis, Theory, Methods \& Applications 811 (1984), 1373-1381. Zbl0569.35011 MR0764917

3. Bardi, M. \& LiO, F. D., On the Strong Maximum Principle for Fully Nonlinear Degenerate Elliptic Equations. Archiv der Mathematik 73 (1999), 276-285. Zbl0939. 35038 MR1710100

4. Barles, G., Soner, H. M. \& Souganidis, P. E., Front Propagation and Phase Field Theory. SIAM Journal on Control and Optimization 312 (March 1993), 439-469. Zbl0785. 35049 MR1205984

5. Burger, M., A Framework for the Construction of Level Set Methods for Shape Optimization and Reconstruction. Interfaces and Free Boundaries 5 (2003), 301-329. Zbl1081. 35134 MR1998617

6. Burger, M., Matevosyan, N. \& Wolfram, M.-T., A Level Set Based Shape Optimization Method for an Elliptic Obstacle Problem. Mathematical Models and Methods in Applied Sciences 214 (April 2011), 619-649. Zb11217.35216 MR2795500

7. Capuzzo-Dolcetta, I., A Generalized Hopf-Lax Formula: Analytical and Approximations Aspects. In Geometric Control and Nonsmooth Analysis, F. Ancona, Ed., vol. 76 of Series on Advances in Mathematics for Applied Sciences. World Scientific, 2008, pp. 136-150. Zbl1195. 35105 MR2487751

8. Carlini, E., Falcone, M., Forcadel, N. \& Monneau, R., Convergence of a Generalized FastMarching Method for an Eikonal Equation with a Velocity-Changing Sign. SIAM Journal of Numerical Analysis 466 (2008), 2920-2952. Zb11180 . 65112 MR2439497 
9. Caselles, V., Catté, F., Coll, T. \& Dibos, F., A Geometric Model for Active Contours in Image Processing. Numerische Mathematik 66 (1993), 1-31. Zbl0804.68159 MR1240700

10. Chen, Y.-G., Giga, Y. \& Goto, S., Uniqueness and Existence of Viscosity Solutions of Generalized Mean Curvature Flow Equations. Journal of Differential Geometry 33 (1991), 749-786. Zb10696 . 35087 MR1100211

11. Crandall, M. G., Viscosity Solutions: a Primer. In Viscosity Solutions and Applications, vol. 1660 of Lecture Notes in Mathematics. Springer, 1997, pp. 1-43. Zb10901.49026 MR1462699

12. Crandall, M. G., Ishit, H. \& Lions, P.-L., User's Guide to Viscosity Solutions of Second Order Partial Differential Equations. Bulletin of the American Mathematical Society 271 (July 1992), 1-67. Zb10755.35015 MR1118699

13. Delfour, M. C. \& Zolésio, J.-P., Shapes and Geometries: Metrics, Analysis, Differential Calculus, and Optimization, second ed. Advances in Design and Control. SIAM, 2011. Zbl1251.49001 MR2731611

14. Droske, M. \& Ring, W., A Mumford-Shah Level-Set Approach For Geometric Image Registration. SIAM Journal on Applied Mathematics 666 (2006), 2127-2148. Zbl1112. 49029 MR2262967

15. Eaton, J. W., Bateman, D., Hauberg, S. \& Wehbring, R., GNU Octave version 4.0.0 manual: a high-level interactive language for numerical computations, 2015. https://ww . gnu .org/software/ octave/doc/interpreter/.

16. Evans, L.C., Partial Differential Equations, vol. 19 of Graduate Studies in Mathematics. American Mathematical Society, 1998. Zbl0902.35002 MR1625845

17. Evans, L. C. \& GARIEPY, R. F., Measure Theory and Fine Properties of Functions. Studies in Advanced Mathematics. CRC Press, 1992. Zb10804.28001 MR1158660

18. Evans, L. C. \& Spruck, J., Motion of Level Sets by Mean Curvature, I. Journal of Differential Geometry 333 (1991), 635-681. Zbl0726.53029 MR1100206

19. Falcone, M., Giorgi, T. \& Loreti, P., Level Sets of Viscosity Solutions: Some Applications to Fronts and Rendez-Vous Problems. SIAM Journal on Applied Mathematics 545 (1994), 1335-1354. Zb10808.49029 MR1293102

20. GigA, Y., Surface Evolution Equations: A Level Set Approach, vol. 99 of Monographs in Mathematics. Birkhäuser, 2006. Zbl1096.53039 MR2238463

21. Gilbarg, D. \& Trudinger, N. S., Elliptic Partial Differential Equations of Second Order, vol. 224 of Grundlehren der mathematischen Wissenschaften. Springer, 1983. Zb10562. 35001 MR0737190

22. Helms, L. L., Potential Theory, second ed. Springer, 2014. Zbl1295. 31001 MR3308615

23. Henrot, A. \& Pierre, M., Variation et optimisation de formes: Une analyse géométrique, vol. 48 of Mathématiques \& Applications. Springer, 2005. Zbl1098.49001 MR2512810

24. Kawohl, B. \& Kutev, N., Comparison Principle and Lipschitz Regularity for Viscosity Solutions of Some Classes of Nonlinear Partial Differential Equations. Funkcialaj Ekvacioj 43 (2000), 241-253. Zbl1142.35315 MR1795972

25. Kraft, D., A Hopf-Lax Formula for the Level-Set Equation and Applications to PDE-Constrained Shape Optimisation. In Proceedings of the 19th International Conference on Methods and Models in Automation and Robotics (2014), IEEE Xplore, pp. 498-503.

26. KRAFT, D., The level-set Package for GNU Octave. Octave Forge, 2014-2015. http://octave. sourceforge.net/level-set/.

27. Kraft, D., A Level-Set Framework for Shape Optimisation. PhD thesis, University of Graz, 2015. https://www.domob.eu/research/phd/thesis.pdf.

28. Kraft, D., Measure-Theoretic Properties of Level Sets of Distance Functions. Journal of Geometric Analysis 26 (2016), 2777-2796. http: / dx . doi .org/10.1007/s12220-015-9648-9.

29. Lions, P.-L., Generalized Solutions of Hamilton-Jacobi Equations, vol. 69 of Research Notes in Mathematics. Pitman Advanced Publishing Program, 1982. Zbl0497. 35001 MR0667669

30. Osher, S. J. \& Sethian, J. A., Fronts Propagating with Curvature-Dependent Speed: Algorithms Based 
on Hamilton-Jacobi Formulations. Journal of Computational Physics 79 (1988), 12-49. Zb10659. 65132 MR0965860

31. Rudin, W., Real and Complex Analysis, third ed. McGraw-Hill Book Company, 1987. Zb10925.00005 MR1736644

32. SAntosA, F., A Level-Set Approach for Inverse Problems Involving Obstacles. ESIAM: Control, Optimisation and Calculus of Variations 1 (1996), 17-33. Zb10870.49016 MR1382514

33. Sethinan, J. A., A Fast Marching Level Set Method for Monotonically Advancing Fronts. Proceedings of the National Academy of Sciences 93 (1996), 1591-1595. Zb10852 . 65055 MR1374010

34. Sethinan, J. A. Level Set Methods and Fast Marching Methods: Evolving Interfaces in Computational Geometry, Fluid Mechanics, Computer Vision, and Materials Science, second ed. Cambridge University Press, 1999. Zbl0973.76003 MR1700751

35. Sokolowski, J. \& ZolÉsio, J.-P., Introduction to Shape Optimization: Shape Sensitivity Analysis, vol. 16 of Springer Series in Computational Mathematics. Springer, 1992. Zb10761. 73003 MR1215733

36. Soner, H. M., Motion of a Set by the Curvature of Its Boundary. Journal of Differential Equations 101 (1993), 313-372. Zb10769. 35070 MR1204331

37. Vemuri, B. C., Ye, J., Chen, Y. \& Leonard, C. M., Image Registration via Level-Set Motion: Applications to Atlas-Based Segmentation. Medical Image Analysis 7 (2003), 1-20.

38. YeH, J., Real Analysis: Theory of Measure and Integration, second ed. World Scientific, 2006. Zbl1098. 28002 MR2250344

39. Younes, L., Shapes and Diffeomorphisms, vol. 171 of Applied Mathematical Sciences. Springer, 2010. Zbl1205.68355 MR2656312 